\title{
Continuously Monitoring Alternative Shortest Paths on Road Networks
}

\author{
Lingxiao $\mathrm{Li}^{\ddagger}$, Muhammad Aamir Cheema ${ }^{\ddagger}$, Mohammed Eunus Alis, Hua Lư ${ }^{\dagger}$, David Taniar ${ }^{\ddagger}$ \\ ${ }^{\ddagger}$ Faculty of Information Technology, Monash University, Australia \\ $\S$ Bangladesh University of Engineering and Technology, Bangladesh \\ †Department of People and Technology, Roskilde University, Denmark \\ $\ddagger$ lingxiao.li, aamir.cheema, david.taniar\}@monash.edu, ${ }^{\S}$ eunus@cse.buet.ac.bd, ${ }^{\dagger} l u h u a @ r u c . d k$
}

\begin{abstract}
Modern navigation systems do not only provide shortest paths but also some alternative paths to provide more options to the users. This paper is the first to study the problem of continuously reporting alternative paths for a user traveling along a given path. Specifically, given a path $P$ on which a user is traveling, we continuously report to the user $k$ paths from the user's current location on $P$ to the target $t$. We present several algorithms each improving on the previous based on non-trivial observations and novel optimisations. The proposed algorithms maintain and exploit the previously computed useful information to efficiently update the $k$ alternative paths as the user moves. We provide space and time complexity analysis for each proposed algorithm. We conduct a comprehensive experimental study on large real-world road networks. The results demonstrate that the proposed algorithms are up to several orders of magnitude faster than the straightforward algorithms.
\end{abstract}

PVLDB Reference Format:

L. Li, M. A. Cheema, M. E. Ali, H. Lu, D. Taniar. Continuously Monitoring Alternative Shortest Paths on Road Networks. PVLDB, 13(11): 2243-2255, 2020.

DOI: https://doi.org/10.14778/3407790.3407822

\section{INTRODUCTION}

Given a source $s$ and a target $t$, a shortest path query [28, $31,3,36,7]$ returns the path from $s$ to $t$ with the minimum cost (e.g., distance, travel time). Shortest path queries are of fundamental importance to a wide variety of map-based systems. However, a shortest path may not always match a user's traveling choices and, therefore, modern map-based systems often provide several alternative paths from which the user can choose a path of their choice to travel along.

A snapshot alternative paths query returns top- $k$ alternative paths (including the shortest path) from $s$ to $t$. In contrast, for a user moving on a path $P$ towards the target $t$, a continuous alternative paths query continuously reports top- $k$ alternative paths from the user's current location $s_{i}$ to $t$. A large body of research has focused on snapshot alternative paths queries $[19,5,13,6,15,14,24]$. However, to the

This work is licensed under the Creative Commons AttributionNonCommercial-NoDerivatives 4.0 International License. To view a copy of this license, visit http://creativecommons.org/licenses/by-nc-nd/4.0/. For any use beyond those covered by this license, obtain permission by emailing info@ vldb.org. Copyright is held by the owner/author(s). Publication rights licensed to the VLDB Endowment.

Proceedings of the VLDB Endowment, Vol. 13, No. 11

ISSN 2150-8097.

DOI: https://doi.org/10.14778/3407790.3407822

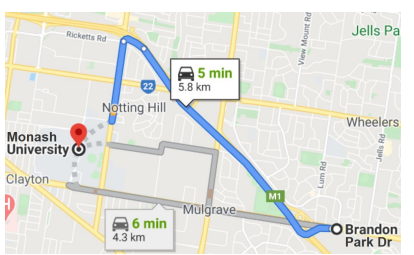

(a)

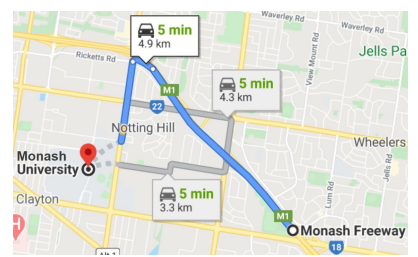

(b)
Figure 1: Alternative paths to Monash University from the user's current location: (a) Brandon Park Drive; (b) Monash Freeway.

best of our knowledge, we are the first to study continuous alternative paths queries.

Motivation. A snapshot alternative paths query provides fixed choices at the start of a user's journey. In contrast, a continuous query goes one step further and continuously reports the alternative paths in real-time as the user travels towards the target. Note that, as the user moves, new interesting alternative paths may appear that were not reported to the user previously and thus giving new options in real-time. Consider the example in Fig. 1 where a user is traveling to Monash University. Fig. 1(a) shows three paths to Monash University when the user is at Brandon Park Drive. Assume that the user is travelling on the shortest path in terms of traveling time (the blue path that goes through M1 - Monash Freeway). As the user enters the Monash Freeway (see Fig. 1(b)), two new alternative paths can be found that have travel time similar to the user's current path. Note that these alternatives did not appear when the user was at Brandon Park Drive. Presenting these new paths gives the user more options to make a decision. Motivated by this, some commercial navigation systems (e.g., Google Maps) continuously report alternative paths to the users as they are moving towards their target.

In addition to enhancing navigation experience, continuously maintaining alternative paths in real time may also help autonomous vehicles, e.g., to reduce/avoid potential congestion [9] and to ensure flexible and safe driving. Limiting the alternative choices results in a constrained search space, forcing an autonomous vehicle to operate less flexibly. For example, if alternative paths are not updated continuously and timely, it may reduce an autonomous vehicle's maneuver time and thus affects its driving safety.

What are "good" alternative paths? A shortest path is well-defined as it corresponds to the path with the minimum cost. In contrast, a "good" alternative path is not welldefined. Intuitively, a set of $k$ alternative paths is "good" if a user finds these paths to be meaningful/natural (e.g., a path does not entail unnecessary detours) and significantly differ- 
ent from each other. Since "goodness" of alternative paths is mostly subjective, existing studies for snapshot alternative paths use different approaches to define and compute top- $k$ alternative paths using different intuitive ideas. However, due to the subjective nature of "goodness", there is no consensus on which of these techniques generates paths perceived better by users. Therefore, before we can propose techniques to continuously monitor alternative paths, we must choose one of the existing approaches to define the top- $k$ alternative paths.

As to be detailed in Section 2.2, existing techniques for snapshot alternative paths fall into three broad categories: 1) plateaus-based techniques $[19,5,20,29,17]$; 2) penaltybased techniques [13, 6, 8, 21]; and 3) dissimilarity-based techniques [15, 14, 24]. Many commercial systems (e.g., Google Maps and TomTom) also generate alternative paths. However, these techniques are proprietary/confidential. Consequently, the research community and open-source community are unable to use these techniques in their implementation/systems. A recent user study [22] conducted on the Melbourne road network shows that the above mentioned techniques (including Google Maps) all return results with comparable path quality. Therefore, any of the existing techniques can be used to define top- $k$ alternative paths. In this work, we choose to use the plateaus-based approach to generate alternative paths for the following reasons: 1) among the published techniques, the plateaus-based approach is arguably the most popular approach and has been used in several commercial and open-source systems (e.g., Cotares Limited $^{1}$ and GraphHopper ${ }^{2,3}$ ); 2) the paths generated using plateaus-based approach are guaranteed to be local optimal [5]; and 3) the computational cost to generate alternative paths using plateaus-based techniques is significantly smaller than the other techniques (e.g., generating dissimilarity-based alternative paths is NP-hard [14]).

Challenges and Contributions. As to be detailed in Section 2.2, the plateaus-based approach needs to create a forward shortest path tree $T_{f}$ (rooted at the user's current location) and a backward shortest path tree $T_{b}$ (rooted at target $t$ ), and to join the two trees. A straightforward solution to continuously report $k$ alternative paths is to recompute $T_{f}$ whenever the user moves. However, this is computationally expensive especially for large road networks. Also, it is non-trivial to efficiently update $T_{f}$ instead of recomputing it. Even if the tree can be efficiently updated, joining $T_{f}$ and $T_{b}$ is not cheap since it requires traversing the two trees. Thus, there is a need to design techniques that can materialize the information computed earlier and can exploit this to efficiently update the $k$ alternative paths without the need to update or join the two trees. We summarize our contributions below.

- To the best of our knowledge, we are the first to study the problem of continuously monitoring $k$ alternative paths.

- We design novel techniques that exploit the fact that the backward tree $T_{b}$ does not change as the user moves on the path. We categorise the vertices of $T_{b}$ into familiar and unfamiliar vertices and use these to define maxdepth for each vertex which is an upper bound on the maximum length of a plateau. Based on careful observations, we present techniques to efficiently update maxdepth of the

\footnotetext{
${ }^{1}$ http://www. cotares.com/routing/

${ }^{2}$ GraphHopper is an open-source routing library and has joined OpenStreetMap Foundation as a gold member.

${ }^{3}$ https : //www . graphhopper . com/blog/2016/01/19/ alternative-roads-to-rome/
}

affected vertices. Finally, we present a novel idea to accessing the vertices in descending order of their maxdepth values to efficiently compute the results.

- We provide an extensive experimental study on several real-world road networks which demonstrates that our techniques are up to several orders of magnitude faster than the straightforward approaches. We also demonstrate the effectiveness of our proposed optimisations.

\section{PRELIMINARIES}

\subsection{Problem Definition}

Similar to many existing works $[2,37,1]$, we assume that the edges in the road network are bidirectional. However, our techniques can be trivially extended for directed networks. Let $G=(\mathcal{V}, \mathcal{E})$ be a graph representing the road network where $\mathcal{V}$ is the set of vertices and $\mathcal{E}$ is the set of edges. Each edge $e=(u, v)$ in $\mathcal{E}$ is assigned a weight $w(e)>0$, e.g., distance or travel time etc. Given two vertices $s, t \in \mathcal{V}$, a shortest path $s p(s, t)$ is a sequence of edges $\left(e_{1}, e_{2}, \ldots, e_{n}\right)$ that connects $s$ to $t$ such that $\sum_{i=1}^{n} w\left(e_{i}\right)$ is minimized. The shortest distance between $s$ and $t$, denoted as $d(s, t)$, is the total weight of $s p(s, t) . d(s, u, t)$ denotes $d(s, u)+d(u, t)$ and $s p(s, u, t)$ denotes $s p(s, u) \oplus s p(u, t)$ where $\oplus$ is the concatenation operation. Table 1 lists the frequently used symbols throughout the paper.

Table 1: Frequently used notations

\begin{tabular}{|l|l|}
\hline Notation & Description \\
\hline \hline$e(u, v)$ & an edge between vertices $u$ and $v$ \\
$w(u, v)$ & weight of the edge $e(u, v)$ \\
$T_{f}$ & forward shortest path tree rooted at $s$ \\
$T_{b}$ & backward shortest path tree rooted at $t$ \\
$p l(u, v)$ & plateau with source and target ends $u, v$ \\
$s p(u, v)$ & the shortest path between $s$ and $t$ \\
$d^{E}(u, v)$ & Euclidean distance between $u$ and $v$ \\
$d(u, v)$ & shortest distance between $s$ and $t$ \\
$d(u, v, w)$ & $d(u, v)+d(v, w)$ \\
$P$ & a user's traveling path $\left\langle s_{1}, s_{2}, s_{3}, \ldots, s_{|p|}\right\rangle$ \\
$d^{P}\left(s_{i}, s_{j}\right)$ & length of the segment $\left\langle s_{i}, \cdots, s_{j}\right\rangle$ of $P$ \\
$L^{k}$ & length of the $k$-th longest plateau \\
$d v_{v}^{s_{i}}$ & deviation vertex of $v$ when user is at $s_{i}$ \\
$v . d e p t h[u]$ & depth of $v$ w.r.t. $u$ \\
$v . m a x d e p t h$ & maximum depth of a vertex $v$ \\
\hline
\end{tabular}

\subsubsection{Plateaus-based Alternate Paths}

Given a source $s$ and a target $t$, plateaus are generated by joining the forward shortest path tree $T_{f}$ and the backward shortest path tree $T_{b}$ where $T_{f}$ is rooted at the source vertex $s$ and $T_{b}$ is rooted at the target vertex $t$. For simplicity of presentation, similar to many existing works $[4,10]$, we assume that the shortest path between any two vertices is unique implying that each shortest path tree is unique. The common branches ${ }^{4}$ of these trees are called plateaus. We use $p l(u, v)$ to denote a plateau where $v$ is closer to the target $t$ (called the target end) and $u$ is closer to $s$ (called the source end). The length of a plateau $p l(u, v)$ is $d(u, v)$, the shortest distance between $u$ and $v$. In Fig. 2(a), assume that source is $s_{1}$ and target is $s_{5}$. Fig. 2(c) and Fig. 2(d) show the forward and backward shortest path trees, respectively. The plateaus (common branches) are shown using same colors in

\footnotetext{
${ }^{4}$ Let $u$ and $v$ be two nodes such that $v$ is an ancestor of $u$ in a tree. We use branch to refer to a chain of edges connecting $u$ and $v$.
} 


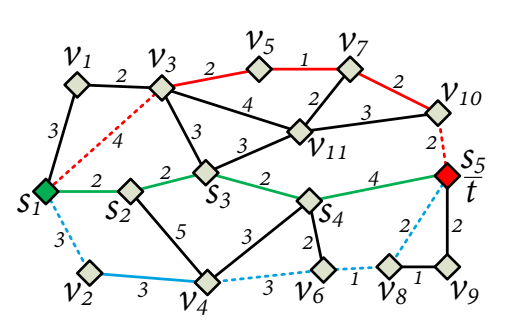

(a) user is at $s_{1}$

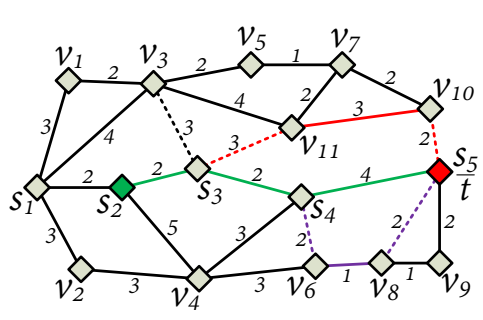

(b) user is at $s_{2}$

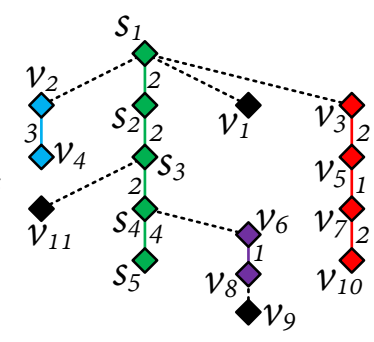

(c) $T_{f}$ with root $s_{1}$

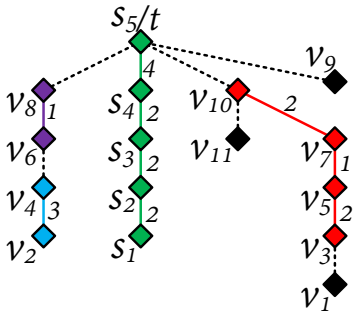

(d) $T_{b}$ with root $s_{5}$

Figure 2: A sample road network where user's path $P=\left\langle s_{1}, s_{2}, \cdots, s_{5}\right\rangle$. Top-3 plateaus when user is at $s_{1}$ are $p l\left(s_{1}, s_{5}\right), p l\left(v_{3}, v_{10}\right)$ and $p l\left(v_{2}, v_{4}\right)$.

both trees except the black vertices (that represent common branches/plateaus with length 0 , i.e., no edge containing such a vertex is common in the two trees). For example $\left\langle v_{3}, v_{5}, v_{7}, v_{10}\right\rangle$ is a plateau (represented as $\left.p l\left(v_{3}, v_{10}\right)\right)$ with length 5 (shown in red). $v_{3}$ is the source end and $v_{10}$ is the target end of this plateau. Other plateaus are $p l\left(s_{1}, s_{5}\right)$ with length 10 (shown green), $p l\left(v_{2}, v_{4}\right)$ with length 3 (shown blue) and $p l\left(v_{6}, v_{8}\right)$ with length 1 (shown purple).

Given a plateau $p l(u, v)$, the alternative path from $s$ to $t$ based on $p l(u, v)$ is $s p(s, u) \oplus p l(u, v) \oplus s p(v, t)$. In Fig. 2(a), the alternative path using $p l\left(v_{3}, v_{10}\right)$ is $\left\langle s_{1}, v_{3}, v_{5}, v_{7}, v_{10}, t\right\rangle$. Note that $p l(u, v)$ represents the shortest path from $u$ to $v$, i.e., $s p(u, v)$. Next, we briefly describe some properties of the plateaus. Proofs are straightforward and omitted.

Property 1. A plateau pl $(u, v)$ is the overlap of $\operatorname{sp}(s, v)$ and $\operatorname{sp}(u, t)$.

PROPERTY 2. Let $u$ be a descendent of $v$ in the backward tree $T_{b}$. Vertices $u$ and $v$ are on the same plateau iff $d(s, u)+$ $d(u, v)=d(s, v)$.

Property 3. For any plateau $\mathrm{pl}(u, v)$, we have $d(s, u)+$ $d(u, t)=d(s, v)+d(v, t)$, i.e., $d(s, u, t)=d(s, v, t)$.

PROPERTY 4. Two plateaus cannot intersect each other (because each shortest path tree is unique).

In Fig. 2(a) where source is $s_{1}$ and target is $s_{5}, p l\left(v_{3}, v_{10}\right)$ is the overlap of $s p\left(s_{1}, v_{10}\right)$ and $s p\left(v_{3}, s_{5}\right)$ (Property 1). Also, $d\left(s_{1}, v_{3}\right)+d\left(v_{3}, v_{10}\right)=d\left(s_{1}, v_{10}\right)$ (Property 2$)$ and $d\left(s_{1}, v_{3}\right)+$ $d\left(v_{3}, s_{5}\right)=d\left(s_{1}, v_{10}\right)+d\left(v_{10}, s_{5}\right)$ (Property 3$)$.

\subsubsection{Continuous Alternative Paths}

Let $P=\left\langle s_{1}, s_{2}, \cdots, s_{|P|}\right\rangle$ be a simple path on which a user is travelling where $s_{|P|}$ is the target vertex, i.e., $t=$ $s_{|P|}$. Hereafter, we use $s_{|P|}$ and $t$ interchangeably. We use $P\left(s_{i}, s_{j}\right)$ to denote the user's path from $s_{i}$ to $s_{j}$ for $i<$ $j \leq|P|$, i.e., $P\left(s_{i}, s_{j}\right)=\left\langle s_{i}, \cdots, s_{j}\right\rangle$. Furthermore, we use $d\left(s_{i}, s_{j}\right)$ to denote the shortest distance between $s_{i}$ and $s_{j}$ and $d^{P}\left(s_{i}, s_{j}\right)$ as the path distance from $s_{i}$ to $s_{j}$ as the user travels along the path $P$, i.e., $d^{P}\left(s_{i}, s_{j}\right)=\sum_{k=i}^{j-1} w\left(s_{k}, s_{k+1}\right)$. In this paper, we continuously report $k$ alternative paths for each location of the user $s_{i} \in P$. In real world applications, the users may not want an alternative path which is significantly larger than the path length from $s_{i}$ to the target $s_{|P|}$. This can be controlled using a user defined parameter $\epsilon \geq 1$ such that any path reported to the user must have length at most equal to $d^{P}\left(s_{i}, s_{|P|}\right) \times \epsilon$. To formalize this, we define valid plateaus.

Definition 1. Valid plateau. Let $P=\left\langle s_{1}, \cdots, s_{|P|}\right\rangle$ be the path a user is traveling on and $s_{i}$ be the user's current location. Given a user-defined parameter $\epsilon \geq 1$, a plateau $p l(u, v)$ is valid if the alternative path generated by it has length at most $d^{P}\left(s_{i}, t\right) \times \epsilon$, i.e., $d\left(s_{i}, v, t\right) \leq d^{P}\left(s_{i}, t\right) \times \epsilon$.
Since each plateau is unique and generates a unique alternative path, for simplicity, we use plateau to refer to an alternative path whenever clear by context. Our techniques report $k$ longest plateaus (top- $k$ plateaus) at each location $s_{i}$ and these plateaus can be used to easily generate $k$ alternative paths.

Definition 2. Top-k Plateaus (TKP) query. Given the current location $s_{i}$ of a user on the path $P$, an integer $k$ and a distance upper bound parameter $\epsilon \geq 1$, the TKP query $Q=\left(s_{i}, P, k, \epsilon\right)$ returns $k$ longest valid plateaus w.r.t. $s_{i}$ and $t$.

Definition 3. Continuous Top-k Plateaus (CTKP) query. Given a positive integer $k$, a distance upper bound parameter $\epsilon \geq 1$ and the user's path $P=\left\langle s_{1}, s_{2}, s_{3}, \ldots, s_{|p|}\right\rangle$, the CTKP query $Q=(k, \epsilon, P)$ query continuously returns the top- $k$ valid plateaus $R_{i}$ for each vertex $s_{i}$ on $P$.

Whenever clear by context, hereafter, we use plateau to refer to a valid plateau. Consider the example in Fig. 2(a). Suppose that $\epsilon=1.3$ and the user is traveling on the shortest path from $s_{1}$ to $s_{5} / t$ (shown green). When the user is at $s_{1}$, the top-3 plateaus are $p l\left(s_{1}, s_{5}\right), p l\left(v_{3}, v_{10}\right)$ and $p l\left(v_{2}, v_{4}\right)$ with lengths 10,5 and 3 , generating three paths shown in green, red and blue lines in Fig. 2(a), respectively. When the user is at $s_{2}$, the top-3 plateaus can be obtained by joining the forward shortest path tree $T_{f}$ rooted at $s_{2}$ (see Fig. 3(a)) and $T_{b}$ (Fig. 2(d)). The top-3 valid plateaus at $s_{2}$ are $p l\left(s_{2}, s_{5}\right), p l\left(v_{11}, v_{10}\right)$ and $p l\left(v_{6}, v_{8}\right)$ with lengths 8,3 and 1 , respectively, generating three alternative paths shown in green, red and purple lines in Fig. 2(b). Note that $p l\left(v_{3}, v_{5}\right)$ is also a plateau with length 2 . However, since $\epsilon=1.3$, the plateau $\operatorname{pl}\left(v_{3}, v_{5}\right)$ is invalid because the path generated by it $\left\langle s_{2}, s_{3}, v_{3}, v_{5}, v_{7}, v_{10}, s_{5}\right\rangle$ has length $12>\left(d^{P}\left(s_{2}, t\right) \times \epsilon=\right.$ $8 \times 1.3=10.4$ ). Thus, this plateau (and consequently the path generated by it) is ignored.

Some existing studies aim to further improve the quality of reported alternative paths by employing additional filtering/ranking criteria (e.g., uniformly bounded stretch [5]). To this end, our techniques can be immediately used to efficiently monitor top- $m(m>k)$ plateaus and the paths generated using these $m$ plateaus can be refined to obtain the top- $k$ alternative paths satisfying the additional criteria.

\subsection{Related Work}

While various other types of continuous queries have received significant attention $[12,11,26,30]$, to the best of our knowledge, we are the first to study continuous top$k$ alternative paths queries. For the snapshot alternative 
paths, other than the plateaus-based techniques, there are two broad categories of the existing techniques. Penaltybased techniques $[6,13]$ iteratively compute shortest paths and, after each iteration, apply a penalty to each edge on the shortest path found in the previous iteration by increasing its edge weight. Further constraints can be applied to prune the retrieved shortest paths if they do not meet a user's requirement (e.g., are longer than a given threshold). The algorithm terminates when $k$ paths satisfying the constraints are found. While the $k$ paths returned by the penalty-based method may be quite similar to each other, the plateaus-based paths are naturally dissimilar to each other (as explained in [22]). Dissimilarity-based techniques $[15,14,24]$ specifically aim to select dissimilar paths by defining a function to measure the dissimilarity between a path $p$ and a given set of paths $P$. The goal is to iteratively add paths $p$ in ascending order of their lengths to the current result set $P$ if the dissimilarity between $p$ and $P$ is greater than a threshold. The problem is shown to be NP-hard [14] and the existing studies mainly focus on approximate solutions. A recent user study [22] discusses more details of the existing studies on computing snapshot alternative paths and provides a user study comparing the path quality of these approaches.

\section{TECHNIQUES}

We first briefly describe a straightforward algorithm based on tree joins (TJ) (Section 3.1). Then, we propose an algorithm based on a top down (TD) traversal of the backward tree (Section 3.2). Finally, built on two novel optimisations, we present our main algorithms named: depth-aware top down (DA-TD) algorithm (Section 3.3) and depth-aware best first (DA-BF) algorithm (Section 3.4). Initially (when user is at $s_{1}$ ), all algorithms compute the top- $k$ plateaus by joining $T_{f}$ and $T_{b}$. We focus on how each algorithm updates the results for each subsequent location $s_{i}$ of the user.

\subsection{Tree-Join Algorithm}

For each location of the user $s_{i}$ on the path $P$, the top$k$ plateaus can be obtained by joining the forward shortest path tree $T_{f}$ rooted at $s_{i}$ and the backward shortest path tree $T_{b}$ rooted at the target $s_{|P|}=t$. Tree-Join algorithm exploits the fact that the $T_{b}$ does not change because the target vertex remains the same. Therefore, for each location $s_{i}$, the algorithm creates $T_{f}$ and joins it with $T_{b}$ to obtain the top- $k$ plateaus. The tree $T_{f}$ is created using Dijkstra's algorithm [16]. The two trees $T_{f}$ and $T_{b}$ can be joined in time linear to the size of the two trees using backward pointers [19]. Next, we present a pruning rule to prune the subtree rooted at $v$.

PRUNing Rule 1. Let $s_{i}$ be the user's current location and $v$ be a node in the backward tree $T_{b}$ such that $d\left(s_{i}, v\right)+$ $d(v, t)>d^{P}\left(s_{i}, t\right) \times \epsilon$. The subtree rooted at $v$ cannot have any valid plateau for the user's location $s_{i}$.

Proof. Let $u$ be any descendant node of $v$ in $T_{b}$. We show that $u$ cannot be on any valid plateau by showing $d\left(s_{i}, u\right)+d(u, t)>d^{P}\left(s_{i}, t\right) \times \epsilon$. Due to the triangle inequality, $d\left(s_{i}, u\right) \geq d\left(s_{i}, v\right)-d(u, v)$. Also, since $u$ is a descendant of $v$ in $T_{b}$ with root $t$, we have $d(u, t)=d(u, v)+d(v, t)$. Thus, $d\left(s_{i}, u\right)+d(u, t) \geq d\left(s_{i}, v\right)-d(u, v)+d(u, v)+d(v, t)$. Hence, $d\left(s_{i}, u\right)+d(u, t) \geq d\left(s_{i}, v\right)+d(v, t)>d^{P}\left(s_{i}, t\right) \times \epsilon$.

Consider our running example in Fig. 2 where $\epsilon=1.3$. Fig. 3 shows $T_{f}$ and $T_{b}$ when the user is at $s_{2}$ and $d^{P}\left(s_{2}, t\right)=$ 8. The subtree rooted at $v_{7}$ in Fig. 3(b) can be pruned because $d\left(s_{2}, v_{7}\right)+d\left(v_{7}, t\right)=7+4=11$ is greater than $d^{P}\left(s_{2}, t\right) \times \epsilon=8 \times 1.3=10.4$.

During the construction of $T_{f}$, Dijkstra's algorithm prunes a vertex $v$ that satisfies this condition. Note that $d\left(s_{i}, v\right)$ is known to the Dijkstra's algorithm and $d(v, t)$ can be obtained in $O(1)$ using the backward tree $T_{b}$.

Complexity analysis: We use $E$ to denote the number of edges in the graph. As is typically the case, we assume that the road network is a connected graph, i.e., the space taken by input graph is $O(E)$. Thus, the space complexity is $O(E)$ because the size of each tree is bounded by $O(E)$. The construction of the shortest path tree by Dijkstra'a algorithm is bounded by $O(E \log V)$ where $V$ is the number of vertices in the tree. The two trees can be joined in time linear to the number of vertices in the two trees [19]. Thus, the total time complexity is $O(E \log V)$.

\subsection{Top Down Tree Traversal Algorithm}

One possible approach to improve the performance of the tree-join algorithm is to design techniques to efficiently update $T_{f}$ as the user moves, instead of recomputing $T_{f}$. Firstly, this is non-trivial and, to the best of our knowledge, there does not exist any technique that can efficiently update a shortest path tree when the source node is changed. Furthermore, even if a technique is designed to efficiently update $T_{f}$, this tree must still be joined with $T_{b}$ to obtain the plateaus, which is inefficient. Therefore, instead of trying to efficiently update or partially update $T_{f}$, we look at the problem from a fresh perspective and propose an algorithm that traverses the backward tree $T_{b}$ in a top down fashion to compute the top- $k$ plateaus. A key strength of this tree traversal algorithm is that we can exploit the computed information for the user's future locations.

The key idea is to traverse the backward tree $T_{b}$ in a top down fashion. For each accessed vertex $v$, the algorithm finds the plateau $p l(u, v)$ for which $v$ is the target end. Recall that a plateau $p l(u, v)$ is the overlap of $s p\left(s_{i}, v\right)$ and $s p(u, t)$ (Property 1). Therefore, to find the plateau with the target end $v$, we get $s p\left(s_{i}, v\right)$ by issuing a shortest path query from $s_{i}$ to $v$. Then, we check the overlap of $s p\left(s_{i}, v\right)$ with the subtree rooted at $v$ in $T_{b}$ to obtain the plateau. We illustrate this using an example.

Consider the example in Fig. 3(b) that shows the backward tree $T_{b}$ for our running example where the user's current location is $s_{2}$. Suppose that the top-down traversal has accessed the vertex $v_{8}$ and we want to find the plateau with $v_{8}$ as the target end, i.e., $p l\left(u, v_{8}\right)$. The algorithm issues a shortest path query from $s_{2}$ to $v_{8}$ which returns $s p\left(s_{2}, v_{8}\right)=\left\langle s_{2}, s_{3}, s_{4}, v_{6}, v_{8}\right\rangle$. The overlap of this path with the subtree of $v_{8}$ is $\left\langle v_{6}, v_{8}\right\rangle$ which is a plateau with length 1 (the purple vertices in Fig. 3).

To efficiently obtain the plateau (i.e., overlap of $s p\left(s_{i}, v\right)$ and the subtree rooted at $v$ ), we simply need to find the first vertex $u$ on $s p\left(s_{i}, v\right)$ such that $v$ is the ancestor of $u$ in $T_{b}$. At the first timestamp (i.e., when user is at $s_{1}$ ), we use the interval-based labelling scheme $[32,33]$ to label all vertices in $T_{b}$, which allows checking ancestor-descendent relationship for any two vertices in $O(1)$. Since $T_{b}$ does not change, the labelling remains valid for all future locations.

\subsubsection{Pruning Rules}

Recall that Pruning Rule 1 prunes the subtree rooted at $v$ for the current location $s_{i}$. The next pruning rule shows that the subtree can, in fact, be pruned for all future locations of the user on the path $P$. 


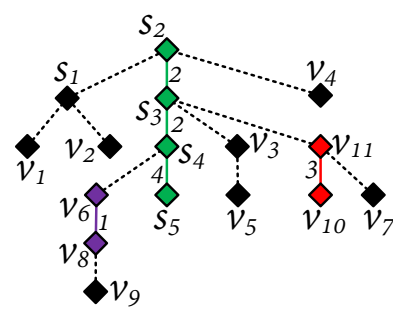

(a) $T_{f}$ at $s_{2}$

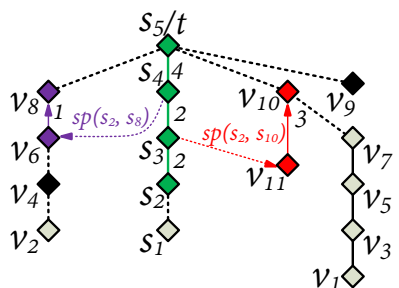

(b) Top down traversal of $T_{b}$
Figure 3: Illustration of the tree-join and topdown algorithms with user at $s_{2}$. Grey vertices are pruned. Vertices with the same color form a plateau.

PRUNing Rule 2. Let $v$ be a node in the backward tree $T_{b}$ such that $d\left(s_{i}, v\right)+d(v, t)>d^{P}\left(s_{i}, t\right) \times \epsilon$. The subtree rooted at $v$ cannot have any valid plateau for any $s_{j}$ on $P$ where $j \geq i$.

Proof. Let $u$ be any descendant node of $v$ in $T_{b}$. The Pruning Rule 1 shows that $d\left(s_{i}, u\right)+d(u, t)>d^{P}\left(s_{i}, t\right) \times \epsilon$. We now show that for any $s_{j}$ on $P$ s.t. $j \geq i, d\left(s_{j}, u\right)+$ $d(u, t)>d^{P}\left(s_{j}, t\right) \times \epsilon$, i.e., $u$ cannot be on any valid plateau for $s_{j}$. Let $d^{P}\left(s_{i}, s_{j}\right)$ be the distance between $s_{i}$ and $s_{j}$ along the path $P$. Note that $d^{P}\left(s_{i}, t\right)=d^{P}\left(s_{i}, s_{j}\right)+d^{P}\left(s_{j}, t\right)$. Thus $d\left(s_{i}, u\right)+d(u, t)>d^{P}\left(s_{i}, t\right) \times \epsilon$ can be written as $d\left(s_{i}, u\right)+d(u, t)>\epsilon \times\left(d^{P}\left(s_{i}, s_{j}\right)+d^{P}\left(s_{j}, t\right)\right)$. Subtracting $d^{P}\left(s_{i}, s_{j}\right)$ on both sides gives us $d\left(s_{i}, u\right)-d^{P}\left(s_{i}, s_{j}\right)+$ $d(u, t)>(\epsilon-1) \times d^{P}\left(s_{i}, s_{j}\right)+\epsilon \times d^{P}\left(s_{j}, t\right)$. Since $d^{P}\left(s_{i}, s_{j}\right) \geq$ $d\left(s_{i}, s_{j}\right)$, due to the triangle inequality, we have $d\left(s_{j}, u\right) \geq$ $d\left(s_{i}, u\right)-d\left(s_{i}, s_{j}\right) \geq d\left(s_{i}, u\right)-d^{P}\left(s_{i}, s_{j}\right)$. Thus, we have $d\left(s_{j}, u\right)+d(u, t)>(\epsilon-1) \times d^{P}\left(s_{i}, s_{j}\right)+\epsilon \times d^{P}\left(s_{j}, t\right)$. Since $\epsilon \geq$ 1 , the right side of the above inequality is at least $d^{P}\left(s_{j}, t\right) \times$ $\epsilon$. Thus, we have $d\left(s_{j}, u\right)+d(u, t)>\epsilon \times d^{P}\left(s_{j}, t\right)$.

At the beginning (i.e., at $s_{1}$ ) when the backward tree $T_{b}$ is computed, we store $d(v, t)$ for each vertex $v$ in $T_{b}$. Thus, $d(v, t)$ can be obtained in $O(1)$. However, $d\left(s_{i}, v\right)$ may not be known when the user moves to $s_{i}$. Instead, we use a lower bound (e.g., Euclidean distance $d^{E}\left(s_{i}, v\right)$ ) to prune the subtree as stated in the next pruning rule.

Pruning Rule 3. Let $v$ be a node in $T_{b}$ where $d^{E}\left(s_{i}, v\right)$ $+d(v, t)>d^{P}\left(s_{i}, t\right) \times \epsilon$. The subtree of $v$ cannot have any valid plateau for every $s_{j}$ on $P$ where $j \geq i$.

The proof is straightforward and is omitted. The next pruning rule prunes the subtree of $v$ by using the length of $k$-th longest plateau found so far by the algorithm.

PRUNing Rule 4. Let $s_{i}$ be a user's current location and $L^{k}$ be the length of the $k$-th longest plateau found so far by the algorithm. The subtree rooted at $v$ cannot contain any top- $k$ plateau for $s_{i}$ if $L^{k}+d(v, t)>d^{P}\left(s_{i}, t\right) \times \epsilon$.

Proof. Assume that a plateau $p l(u, x)$ is longer than $L^{k}$ where both $u$ and $x$ are in the subtree rooted at $v$. We show that the plateau is invalid, i.e., $d(s, x)+d(x, t)>d^{P}\left(s_{i}, t\right) \times$ $\epsilon$. Since $x$ is a node in the subtree of $v$ in the backward tree $T_{b}$ rooted at $t$, we have $d(x, t) \geq d(v, t)$. So, we have $d(s, x)+d(x, t) \geq d(s, x)+d(v, t)$. Also, if $p l(u, x)$ is longer than $L^{k}$, we have $d(s, x)>L^{k}$ (because $p l(u, x) \subseteq s p(s, x)$ according to Property 1$)$. Thus, we have $d(s, x)+d(x, t)>$ $L^{k}+d(v, t)>d^{P}\left(s_{i}, t\right) \times \epsilon$.

Let maxleafdist $(v)$ be the distance from a vertex $v$ to the furthest leaf in the subtree rooted at $v$. The next pruning rule prunes the subtree using maxleafdist $(v)$.

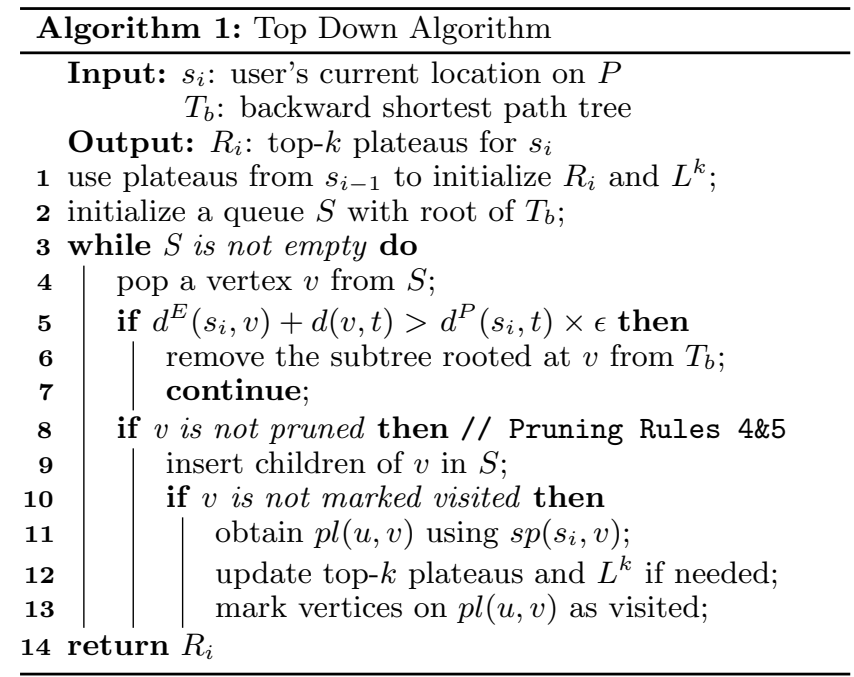

PRUNING Rule 5. The subtree rooted at $v$ cannot contain a top- $k$ plateau if maxleafdist $(v)<L^{k}$.

Proof. Recall that each plateau $p l(u, v)$ is the overlap of $\operatorname{sp}\left(s_{i}, v\right)$ and the subtree rooted at $v$. Hence, the length of any plateau $p l(u, v)$ is bounded by maxleafdist $(v)$. Thus, the length of $p l(u, v)$ is smaller than $L^{k}$.

\subsubsection{Algorithm}

Algorithm 1 details how to update the top- $k$ plateaus when a user moves from $s_{i-1}$ to $s_{i}$. First, we use the top- $k$ plateaus for $s_{i-1}$ and check which of these are still plateaus for the new location $s_{i}$. Specifically, $u$ and $v$ are on the same plateau iff $d\left(s_{i}, u\right)+d(u, t)=d\left(s_{i}, v\right)+d(v, t)$ (Property 2). Hence, we issue two shortest path queries from $s_{i}$ to both $u$ and $v$ to check if $p l(u, v)$ is still a plateau or not. Then, we initialize the set of top- $k$ plateaus $R_{i}$ by inserting the valid plateaus in $R_{i}$ and update $L^{k}$ accordingly (set to 0 if $R_{i}$ contains less than $k$ plateaus). Note that we cannot terminate the algorithm even if all $k$ plateaus from $s_{i-1}$ are still the plateaus for $s_{i}$. This is because the length of any of these plateaus or some other plateaus may have increased thus the top- $k$ plateaus may have changed.

The algorithm accesses the tree in a top-down fashion by iteratively accessing nodes from a queue $S$. We apply the pruning rules presented in Section 3.2.1. Specifically, we remove the whole subtree of $v$ from $T_{b}$ if $d^{E}\left(s_{i}, v\right)+$ $d(v, t)>d^{P}\left(s_{i}, t\right) \times \epsilon$ (line 5$)$ because the subtree cannot contain any valid plateau for all future locations of the user. If $v$ cannot be pruned using the pruning rules, we insert its children in $S$ to be accessed in future iterations (lines 8 and 9). We issue a shortest path query from $s_{i}$ to $v$ to obtain the plateau $p l(u, v)$ and update top- $k$ plateaus and $L^{k}$ as needed (lines 11 and 12). In our implementation, we use pruned highway labeling (PHL) [7] to obtain the shortest paths. Each vertex $v^{\prime}$ on $p l(u, v)$ is marked visited (line 13). In each iteration, a vertex which is already visited is ignored (line 10). This ensures that the algorithm does not issue an unnecessary shortest path query to obtain a plateau $p l\left(u, v^{\prime}\right)$ which is contained by a $p l(u, v)$ already found. The algorithm terminates when the queue $S$ becomes empty.

Fig. 3 shows execution of the top-down algorithm when the user is at $s_{2}$. The algorithm first issues a shortest path query to $s_{5}$ and obtains the plateau $p l\left(s_{2}, s_{5}\right)$ with length 8. When $v_{8}$ and $v_{10}$ are accessed, two plateaus $p l\left(v_{6}, v_{8}\right)$ and $p l\left(v_{11}, v_{10}\right)$ are found with length 1 and 3 , respectively. 
The vertices on these plateaus are marked visited and not processed by the algorithm. Plateaus for $v_{4}$ and $v_{9}$ contain only single nodes, i.e., $p l\left(v_{4}, v_{4}\right)$ and $p l\left(v_{9}, v_{9}\right)$ have length 0 . The grey vertices are pruned by the pruning rules. Hence, the algorithm returns $p l\left(s_{2}, s_{5}\right)$ and $p l\left(v_{11}, v_{10}\right)$ as the top-2 plateaus (assuming $k=2$ ).

Complexity Analysis: The space complexity is $O(E+$ $\left.S P_{\text {index }}\right)$ where $O(E)$ is the space for the input graph and $S P_{\text {index }}$ is the size of the shortest path index (depending on which shortest path algorithm is used). Next, we analyse the time complexity. Let $V_{u}^{T D}$ be the total number of unpruned and umarked vertices by the Top-Down (TD) algorithm and $S P_{\text {cost }}$ be the cost of a single shortest path query. Applying each pruning rule takes $O(1)$. Since the algorithm uses a queue, push and pop operations also take $O(1)$. The algorithm processes $O(V)$ vertices in total and issues $V_{u}^{T D}$ shortest path queries. Therefore, the total cost is $O\left(V+V_{u}^{T D} \times\left(S P_{\text {cost }}+\log k\right)\right)$ where $O(\log k)$ is the cost to update the top- $k$ plateaus whenever a new plateau is found.

\subsection{Depth-Aware Top Down Algorithm}

In this section, we present a novel approach that assigns each node $v$ with a maximum depth which guarantees that $p l(u, v)$ cannot be longer than the maximum depth. Furthermore, the depths do not necessarily become invalid as the user moves from $s_{i}$ to $s_{j}$, which allows reusing the previous computations for the user's new locations.

\subsubsection{Observations}

Definition 4. Deviation vertex \& overlapping path. Let $s_{i}$ be the user's current location. The deviation vertex of a vertex $v$ w.r.t. $s_{i}$ is the first vertex after which the shortest path $s p\left(s_{i}, v\right)$ deviates from the user's path $P\left(s_{i}, t\right)$. Formally, the deviation vertex of $v$ is the first vertex $s_{j}$ on $P\left(s_{i}, t\right)$ such that $P\left(s_{i}, s_{j+1}\right)$ is not a sub-path of $s p\left(s_{i}, v\right)$. The path $P\left(s_{i}, s_{j}\right)$ is called the overlapping path of $v$ because this represents the overlap of $s p\left(s_{i}, v\right)$ and $P\left(s_{i}, t\right)$.

The deviation vertex of $v$ w.r.t. the user's location $s_{i}$ is denoted as $d v_{v}^{s_{i}}$ and the overlapping path is denoted as $P\left(s_{i}, d v_{v}^{s_{i}}\right)$. Consider the example in Fig. 2(a) where the path is $P=\left\langle s_{1}, s_{2}, s_{3}, s_{4}, s_{5}\right\rangle$ and the current location of user is $s_{1}$. By the definition, $s_{4}$ is the deviation vertex for vertex $v_{6}$ because the shortest path from $s_{1}$ to $v_{6}$ is $\left\langle s_{1}, s_{2}, s_{3}, s_{4}, v_{6}\right\rangle$ which deviates from the user's path $P$ at vertex $s_{4}$. The overlapping path of $v_{6}$ is $\left\langle s_{1}, s_{2}, s_{3}, s_{4}\right\rangle$. The deviation vertex and overlapping path for $v_{8}$ are the same as those of $v_{6}$. The deviation vertex of $v_{11}$ is $s_{3}$.

The deviation vertex of $v$ can be computed by issuing a shortest path query from $s_{i}$ to $v$. Specifically, when we issue a shortest path query from $s_{i}$ to a vertex $v$ in Algorithm 1 (line 11), for each vertex $x$ on this shortest path $s p\left(s_{i}, v\right)$, we update the deviation vertex of $x$. Next, we show that the deviation vertex of $v$ does not change as long as the user is on the overlapping path of $v$ (i.e., as long as the user has not crossed the deviation vertex). E.g., in Fig. 2(a), the deviation vertex of $v_{11}$ remains $s_{3}$ as long as the user is on the overlapping path $P\left(s_{1}, s_{3}\right)$, i.e., the user has not crossed $s_{3}$. The next lemma formalizes this.

LEMMA 1. Let $d v_{v}^{s_{i}}$ be the deviation vertex of $v$ for the user at location $s_{i}$. For every $s_{j} \in P\left(s_{i}, d v_{v}^{s_{i}}\right), d v_{v}^{s_{i}}=d v_{v}^{s_{j}}$.

Proof. By definition of the deviation vertex, each $s_{j} \in$ $P\left(s_{i}, d v_{v}^{s_{i}}\right)$ lies on the shortest path $s p\left(s_{i}, v\right)$. Therefore, $s p\left(s_{i}, v\right)=s p\left(s_{i}, s_{j}\right) \oplus s p\left(s_{j}, v\right)$ where $\oplus$ is the concatenation operation. Also, $P\left(s_{i}, t\right)=P\left(s_{i}, s_{j}\right) \oplus P\left(s_{j}, t\right)$. Since $s_{j}$ is on the overlapping path, $P\left(s_{i}, s_{j}\right)=s p\left(s_{i}, s_{j}\right)$ which implies $P\left(s_{i}, t\right)=s p\left(s_{i}, s_{j}\right) \oplus P\left(s_{j}, t\right)$. Thus, $s p\left(s_{j}, v\right)$ and the path $P\left(s_{j}, t\right)$ deviate from each other at the same vertex as $s p\left(s_{i}, v\right)$ and the path $P\left(s_{i}, t\right)$ deviate from each other, i.e., $d v_{v}^{s_{i}}=d v_{v}^{s_{j}}$

Hereafter, whenever clear by context, we use $d v_{v}$ to denote the deviation vertex of $v$ w.r.t. the user's current location.

We have established that the deviation vertex of $v$ does not change unless the user crosses it. When the user crosses it, the algorithm may not know the deviation vertex of $v$ unless it issues another shortest path query which passes through $v$ in which case its new deviation vertex is updated and is known to the algorithm. For example, in Fig. 2, when the user crosses $s_{3}$, the deviation vertex of $v_{11}$ is not known to the algorithm unless a shortest path query involving $v_{11}$ is issued. Next, we classify each vertex based on whether its deviation vertex is known to the algorithm or not.

Familiar/unfamiliar vertices. A vertex $v$ for which the algorithm knows its deviation vertex is called a familiar vertex. In contrast, a vertex is called an unfamiliar vertex if our algorithm does not know its deviation vertex. For each familiar vertex $v$, in addition to its deviation vertex $d v_{v}^{s_{i}}$, we also store $d\left(s_{i}, v\right)$. As we show shortly (Lemma 2), this may help obtaining the shortest distances $d\left(s_{j}, v\right)$ for other vertices $s_{j}$ on the path in constant time.

Initially (at location $s_{1}$ ), the algorithm knows the deviation vertex for each vertex in the graph (which is recorded during the construction of $T_{f}$ ). So, all vertices are familiar initially. In Fig. 2(a), the deviation vertex for $v_{5}$ is $s_{1}$ and the deviation vertex for both $v_{6}$ and $v_{8}$ is $s_{4}$. When the user crosses $s_{1}$ and reaches $s_{2}$, the deviation vertex of $v_{5}$ is unknown whereas the deviation vertex of $v_{6}$ and $v_{8}$ remain $s_{4}$. Thus, at $s_{2}$, both $v_{6}$ and $v_{8}$ are familiar vertices whereas $v_{5}$ is an unfamiliar vertex. Assume that, at $s_{2}$, our algorithm needs to issue a shortest path query $s p\left(s_{2}, v_{5}\right)$ which returns the path $\left\langle s_{2}, s_{3}, v_{3}, v_{5}\right\rangle$. As a result, the deviation vertex for all the unfamiliar vertices on this path (i.e., $v_{3}$, and $v_{5}$ ) is updated to be $s_{3}$ and these vertices become familiar vertices.

Next, we show that the shortest distance to any familiar vertex $v$ can be computed in $O(1)$ as long as the user is on its overlapping path.

Lemma 2. Let $v$ be a vertex which became familiar to the algorithm at location $s_{i}$, i.e., the algorithm records $d\left(s_{i}, v\right)$ for $v$. For every vertex $s_{j}$ on the overlapping path $P\left(s_{i}, d v_{v}^{s_{i}}\right)$, $d\left(s_{j}, v\right)=d\left(s_{i}, v\right)-d\left(s_{i}, s_{j}\right)$.

Proof. By definition of deviation vertex and overlapping path, $s_{j}$ is on $s p\left(s_{i}, v\right)$, which implies that $d\left(s_{i}, v\right)=$ $d\left(s_{i}, s_{j}\right)+d\left(s_{j}, v\right)$. Hence, $d\left(s_{j}, v\right)=d\left(s_{i}, v\right)-d\left(s_{i}, s_{j}\right)$.

Using the above lemma, $d\left(s_{j}, v\right)$ can be computed in $O(1)$ because $d\left(s_{i}, v\right)$ is stored by the algorithm and $d\left(s_{i}, s_{j}\right)$ can also be obtained in $O(1)$. Specifically, at the beginning, for each $s_{k}$ on the path $P$, we record $d^{P}\left(s_{1}, s_{k}\right)$. Hence, $d^{P}\left(s_{i}, s_{j}\right)=d\left(s_{i}, s_{j}\right)=d^{P}\left(s_{1}, s_{j}\right)-d^{P}\left(s_{1}, s_{i}\right)$. Note that $d^{P}\left(s_{i}, s_{j}\right)=d\left(s_{i}, s_{j}\right)$ because $s_{j}$ is on the overlapping path.

Next, we present several lemmas to identify the cases when a vertex $v$ and its child $u$ in $T_{b}$ cannot be on the same plateau. Afterwards, we will present techniques that exploit these to update top- $k$ plateaus efficiently.

LEMMA 3. Let $v$ be a vertex that became familiar to the algorithm at location $s_{i}$. Let $u$ be a child of $v$ in $T_{b}$ which is an unfamiliar vertex at $s_{i}$. Vertices $u$ and $v$ cannot be on the same plateau for the user's location $s_{i}$. 
Proof. We prove this by contradiction. Assume $u$ and $v$ are on the same plateau at $s_{i}$. This implies that the shortest path from $s_{i}$ to $v$ must pass through $u$, i.e., $u \in s p\left(s_{i}, v\right)$. Recall that when our algorithm issues a shortest path query $s p\left(s_{i}, x\right)$, each vertex on $s p\left(s_{i}, x\right)$ becomes a familiar vertex. Since $v$ became familiar at $s_{i}$, this implies $v \in s p\left(s_{i}, x\right)$ for some vertex $x$ for which our algorithm had issued a shortest path query. This implies that $s p\left(s_{i}, v\right) \subseteq s p\left(s_{i}, x\right)$. Since $u \in \operatorname{sp}\left(s_{i}, v\right), u \in \operatorname{sp}\left(s_{i}, x\right)$. Thus, $u$ must also be a familiar vertex which is not the case.

Note that the above lemma implies that $u$ and $v$ cannot be on the same plateau if $u$ is unfamiliar but $v$ is familiar. However, this does not imply that $u$ and $v$ must be on the same plateau if $u$ is also familiar. If both $u$ and $v$ are familiar vertices, $u$ and $v$ may or may not be on the same plateau.

LEMMA 4. Let $u$ be a child of $v$ in $T_{b}$. If $u$ is an unfamiliar vertex and $v$ is a familiar vertex, $u$ and $v$ cannot be on the same plateau as long as the user is on the overlapping path of $v$, i.e., user is on $s_{j} \in P\left(s_{i}, d v_{v}^{s_{i}}\right)$.

Proof. If $u$ and $v$ are on the same plateau for a location $s_{j}$, then $s p\left(s_{j}, v\right)$ must pass through $u$. We prove that, for each $s_{j} \in P\left(s_{i}, d v_{v}^{s_{i}}\right), s p\left(s_{j}, v\right)$ does not pass through $u$. Lemma 3 implies that $u$ and $v$ are not on the same plateau when the user is at $s_{i}$, i.e., $s p\left(s_{i}, v\right)$ does not pass through $u$. By definition of deviation vertex, for each $s_{j} \in P\left(s_{i}, d v_{v}^{s_{i}}\right)$, $s p\left(s_{i}, v\right)=s p\left(s_{i}, s_{j}\right) \oplus s p\left(s_{j}, v\right)$. Since $s p\left(s_{i}, v\right)$ does not pass through $u, s p\left(s_{j}, v\right)$ also cannot pass through $u$.

According to the above lemmas, a familiar vertex $v$ and its unfamiliar child $u$ in $T_{b}$ cannot make a plateau as long as the user has not crossed the deviation vertex of $v$. Consider the example in Fig. 4 where a backward tree $T_{b}$ is shown (not related to the running example). Assume that green vertices are familiar and red vertices are unfamiliar. The vertex $v_{2}$ and its child $v_{5}$ cannot be on the same plateau. Likewise, $v_{4}$ and its child $v_{6}$ cannot be on the same plateau.

LemMa 5. Let $u$ be a child of $v$ in $T_{b}$. Let $u$ and $v$ both be familiar vertices at a user's location $s_{i}$. If $u$ and $v$ are not on the same plateau when the user is at $s_{i}$, they cannot be on the same plateau as long as the user is on the overlapping path of $v$, i.e., $s_{j} \in P\left(s_{i}, d v_{v}^{s_{i}}\right)$.

Proof. Since $u$ and $v$ are not on the same plateau when the user is at $s_{i}, s p\left(s_{i}, v\right)$ does not pass through $u$. By definition of deviation vertex, for each $s_{j} \in P\left(s_{i}, d v_{v}^{s_{i}}\right), s p\left(s_{i}, v\right)=$ $s p\left(s_{i}, s_{j}\right) \oplus s p\left(s_{j}, v\right)$. Since $s p\left(s_{i}, v\right)$ does not pass through $u, s p\left(s_{j}, v\right)$ also cannot pass through $u$.

In Fig. 4, assume that $v_{13}$ and $v_{18}$ are not on the same plateau at a location $s_{i}$. They cannot be on the same plateau as long as the user is on the overlapping path of $v_{13}$. In Fig. 4, we use the dashed edges to show all cases where it is guaranteed (by Lemmas 4 and 5) that $v$ and its child $u$ cannot be on the same plateau for each location on the overlapping path of $v$. For the solid edges, the pair of vertices may or may not be on the same plateau.

\subsubsection{Depth and Maximum Depth}

The lemmas presented in Section 3.3.1 can be used to infer the maximum possible length of any plateau $p l(x, v)$ with $v$ as its target end. Below, we introduce the concept of depth and maximum depth to formalize this.

Definition 5. Depth. Let $u$ be a child of $v$ in the backward tree $T_{b}$. The depth of $v$ w.r.t. $u$, denoted as $v$.depth $[u]$, is the maximum possible length of a plateau $p l(x, v)$ that contains both $u$ and $v$ with $v$ as the target end.

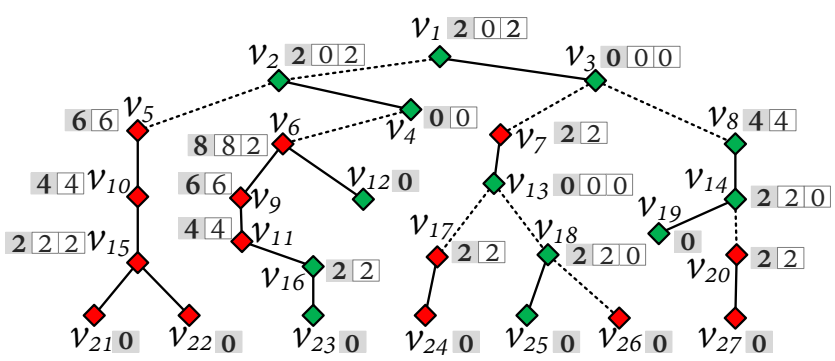

Figure 4: $T_{b}$ with depth arrays and maximum depths. Red vertices are unfamiliar and green vertices are familiar. The dashed edges show that the two vertices cannot be on the same plateau.

Definition 6. Maximum depth. Maximum depth of a node $v$, denoted as $v$.maxdepth, is the maximum possible length of any plateau $p l(x, v)$ with $v$ as the target end. In other words, v.maxdepth $=\max \{v \cdot \operatorname{depth}[u] \mid u \in C\}$ where $C$ denotes all children of $v$ in the backward tree $T_{b}$.

For each non-leaf node $v$ in $T_{b}$, we store its maximum depth along with a depth array that records depths for each of its children. In Fig. 4, depth array for each node is shown along with the maximum depth shown with grey background besides the array. In this example, when the depth array of a node contains two values, the first (resp. second) value in it corresponds to the depth of the node w.r.t. the left (resp. right) child. For simplicity, we assume that each edge has weight 2. As stated earlier, if the algorithm does not yet know whether two nodes are on the same plateau or not, we show the edge in solid line. For $v_{14}$, its depth w.r.t. right child $v_{20}$ is $v_{14}$.depth $\left[v_{20}\right]=0$ because $v_{14}$ and $v_{20}$ cannot be on the same plateau (Lemma 4 ). The depth of $v_{14}$ w.r.t. $v_{19}$ is $v_{14}$.depth $\left[v_{19}\right]=2$ because $v_{14}$ and $v_{19}$ may or may not be on the same plateau but we set depth by assuming that they are on the same plateau (recall depth corresponds to the upper bound on the length of the plateau). The maximum depth of $v_{14}$ is $\max \left(v_{14}\right.$.depth $\left.\left[v_{20}\right], v_{14} \cdot \operatorname{depth}\left[v_{19}\right]\right)=2$. The depth of $v_{3}$ w.r.t. its both children is 0 and its maximum depth is also 0 .

Computing maximum depth and depth array. Before we show how to initialize and continuously update depth arrays and maximum depths of each vertex, we first show how to compute the depth array and the maximum depth of a single vertex $v$ assuming that the maximum depth for each of its children $u$ has been correctly computed, i.e., u.maxdepth is known for each child $u$ of $v$. First, we summarize the observations presented in Section 3.3.1.

Case 1: $v$ is familiar but $u$ is unfamiliar. $u$ and $v$ cannot be on the same plateau (Lemma 4), hence, v.depth $[u]=0$. E.g., $v_{14} \cdot \operatorname{depth}\left[v_{20}\right]=0$ and $v_{3} . \operatorname{depth}\left[v_{7}\right]=0$ in Fig. 4 .

Case 2: $v$ and $u$ both are familiar. In this case, $u$ and $v$ may or may not be on the same plateau. We determine whether $u$ and $v$ are on the same plateau or not. Case 2a: If $u$ and $v$ are not on the same plateau then $v \cdot \operatorname{depth}[u]=0$ (Lemma 5), e.g., $v_{3} . \operatorname{depth}\left[v_{8}\right]=0$. Case 2b: If $u$ and $v$ are on the same plateau then $v \cdot \operatorname{depth}[u]=u \cdot \operatorname{maxdepth}+d(u, v)$, e.g., $v_{8} \cdot \operatorname{depth}\left[v_{14}\right]=v_{14} \cdot$ maxdepth $+d\left(v_{14}, v_{8}\right)=2+2=4$. Case 3: $v$ is unfamiliar. Note that the lemmas presented in Section 3.3.1 do not apply when $v$ is an unfamiliar vertex (regardless of whether $u$ is a familiar vertex or not). Since $v . \operatorname{depth}[u]$ is an upper bound (i.e., maximum length of $p l(x, v)$ with both $u$ and $v$ on it), we assume that $v$ and $u$ are on the same plateau and set $v \cdot \operatorname{depth}[u]=u \cdot \operatorname{maxdepth}+$ $d(u, v)$. E.g., $v_{6} \cdot \operatorname{depth}\left[v_{9}\right]=v_{9} \cdot \operatorname{maxdepth}+d\left(v_{9}, v_{6}\right)=$ 


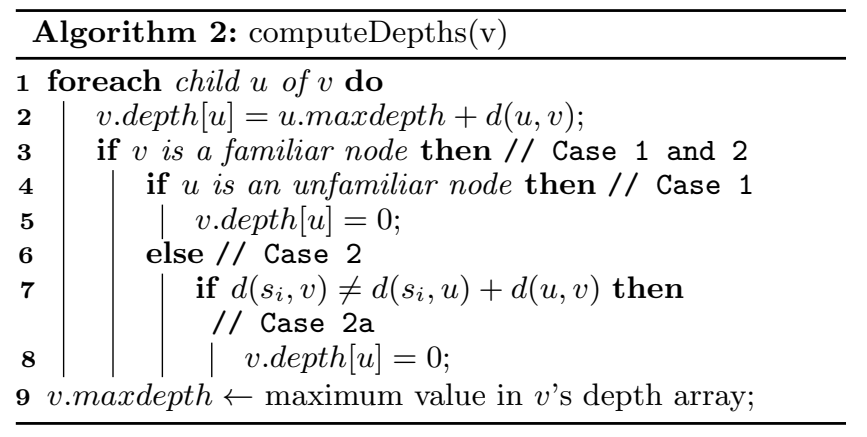

$6+2=8$ and $v_{11} \cdot \operatorname{depth}\left[v_{16}\right]=v_{16} \cdot \operatorname{maxdepth}+d\left(v_{16}, v_{11}\right)=$ $2+2=4$ in Fig. 4 .

Now, we present Algorithm 2 that details how to compute the depth array and maximum depth of $v$ assuming that the maximum depth of each of its children $u$ is known. For each child $u$, we initialize $v \cdot d e p t h[u]=u \cdot \operatorname{maxdepth}+d(u, v)$ assuming Case $2 \mathrm{~b}$ and Case 3 (line 2). Later, we update $v \cdot \operatorname{depth}[u]=0$ if $v$ and $u$ correspond to Case 1 or Case 2a. Specifically, if $v$ is familiar and $u$ is unfamiliar (Case 1), we set $v$.depth $=0$ (line 5). If $v$ and $u$ are both familiar, we check whether $u$ and $v$ are on the same plateau. Recall that $u$ and $v$ are on the same plateau iff $d\left(s_{i}, v\right)=d\left(s_{i}, u\right)+$ $d(u, v)$ (Property 1). Note that $d(u, v)$ is the edge weight and $d\left(s_{i}, x\right)$ for any familiar node $x$ can be computed in $O(1)$ (Lemma 2). Therefore, since $u$ and $v$ are both familiar, we can check in $O(1)$ whether $u$ and $v$ are on the same plateau or not. Otherwise, (Case $2 \mathrm{a}$ ), we update v.depth $[u]=0$ (lines 7 and 8). The maximum depth of $v$ is set to be the maximum of all values in the depth array (line 9).

\subsubsection{Initializing and Maintaining Depth Arrays}

First, we explain how to update depth arrays and maximum depths of affected vertices when a user moves from $s_{i-1}$ to $s_{i}$. Afterwards, we explain how to initialize depth arrays and maximum depths.

Maintaining Depth Arrays. As implied by Lemmas presented in Section 3.3.1, the depth arrays and maximum depths remain valid unless at least one familiar (resp. unfamiliar) vertex becomes unfamiliar (resp. familiar). We handle these two cases as follows.

Case 1: Some familiar vertices become unfamiliar. When the user moves from $s_{i-1}$ to $s_{i}$, the familiar vertices for which the deviation vertex was $s_{i-1}$ become unfamiliar. Let $L$ be the set of such vertices. We update the depth arrays and maximum depths using Algorithm 3. The algorithm updates the depth arrays and maximum depths in a bottom up approach, i.e., the vertices are processed in descending order of level $(v)$ where level of a vertex $v$ is the number of edges between $v$ and the root of the backward tree $T_{b}$. For each vertex $v$, we call Algorithm 2 to recompute its depth arrays and maximum depth. If the maximum depth of $v$ changes, the maximum depth of its parent may also need to be updated (because the depth array of the parent depends on the maximum depths of its children). In that case, we insert the parent of $v$ in $L$ unless it is already in $L$.

Since the algorithm processes the affected vertices in a bottom up fashion, this guarantees that the maximum depth of each child $u$ of $v$ is correctly updated before the depth array of $v$ is computed by Algorithm 2. Note that, in the worst case, the above algorithm requires updating the depth array and maximum depth for each vertex at most once. However, in practice, the total number of vertices for which depth arrays need to be updated is much smaller.

\begin{tabular}{|c|c|}
\hline \multicolumn{2}{|c|}{ Algorithm 3: depthUpdate(L) } \\
\hline & put: $L:$ List of affected vertices \\
\hline & reach $v \in L$ in descending order of level $(v)$ do \\
\hline 2 & $\operatorname{computeDepths}(v)$ \\
\hline 3 & if $v \cdot$ maxdepth changes then \\
\hline 4 & insert parent of $v$ in $L$ if not already present \\
\hline
\end{tabular}

Finally, we describe how to efficiently obtain $L$, the list of vertices that become unfamiliar when a user moves from $s_{i-1}$ to $s_{i}$. This can be efficiently done by maintaining inverted lists for each vertex $s_{i}$ on the path $P$. Specifically, the inverted list for $s_{i}$ contains all vertices for which $s_{i}$ is a deviation vertex. These lists are initialized at $s_{1}$ and are maintained as the algorithm finds the new deviation vertices. Thus, the list of affected vertices when the user moves from $s_{i-1}$ to $s_{i}$ corresponds to the inverted list of $s_{i-1}$.

Case 2: Some unfamiliar vertices become familiar. As stated earlier, when our query processing algorithm issues a shortest path query from $s_{i}$ to a vertex $v$ (e.g., line 11 in Algorithm 1), each vertex $x \in s p\left(s_{i}, v\right)$ becomes a familiar vertex and the deviation vertex of $x$ is updated. To update the depth arrays, we set $L$ by inserting in it each vertex $x \in \operatorname{sp}\left(s_{i}, v\right)$ which was not already a familiar vertex. Then, Algorithm 3 is called with $L$ as the input to update the depth arrays and maximum depths.

Initialization. When the user is at $s_{1}$, we initialize depth arrays and maximum depths of all vertices by calling Algorithm 3 where the input $L$ contains all vertices in $T_{b}$.

\subsubsection{Algorithm}

The Depth-Aware Top Down Algorithm is the same as Algorithm 1 except the following three important differences. 1. Since v.maxdepth is an upper bound on the maximum length of a plateau $p l(x, v)$, we do not need to issue a shortest path query from $s_{i}$ to $v$ to obtain the plateau if $v$.maxdepth is smaller than $L^{k}$, the length of $k$-th longest plateau found so far. Specifically, lines 11 to 13 in Algorithm 1 are not processed if $v$.maxdepth $<L^{k}$.

2. If $v$ is a familiar vertex, Pruning Rule 2 is used at line 5 instead of Pruning Rule 3 , i.e., we use $d\left(s_{i}, v\right)$ instead of the Euclideadn distance $d^{E}\left(s_{i}, v\right)$ because $d\left(s_{i}, v\right)$ can be computed in $O(1)$ for each familiar vertex (Lemma 2).

3. If $v$.maxdepth $\geq L^{k}$, one option to obtain the plateau $p l(x, v)$ is to issue the shortest path query to $v$. However, if $v$ is a familiar vertex, we can get the plateau $p l(x, v)$ without issuing a shortest path query. Specifically, $v$ and its child $u$ can be on the same plateau only if both $u$ and $v$ are familiar. So, we only need to traverse down the branch with a familiar child $u$ such that $u$ and $v$ are on the same plateau which can be confirmed in $O(1)$ (by checking whether $d\left(s_{i}, v\right)=$ $d\left(s_{i}, u\right)+d(u, v)$ as discussed in Section 3.3.2).

Complexity Analysis: The space used by all the depth arrays is $O(V) \leq O(E)$. This is because for each vertex in $T_{b}$, constant space is used in its parent for the depth array. Also, other bookkeeping information (e.g., $\operatorname{dist}\left(s_{i}, v\right)$ and familiar/unfamiliar status) uses constant space per vertex. Thus, the total space complexity is $O\left(E+S p_{\text {index }}\right)$, the same as for the basic top down algorithm.

To analyse time complexity, we first analyse the cost of updating maxdepths. Cost for computeDepths (Algorithm 2) is $O(1)$ since the size of depth array is bounded by maximum out-degree which is a small constant in road networks. Cost of depthUpdate (L) (Algorithm 3) is $O\left(V_{L}\right)$ where $V_{L} \leq V$ is the number of vertices with updated maxdepths. Thus, the total cost to maintain maxdepths is 
$O\left(V_{L}\right)$ which is bounded by $O(V)$. Each pruning rule takes $\mathrm{O}(1)$. Let $V_{u}^{D A}$ be the number of unpruned and umarked vertices for the depth-aware (DA) algorithm. The algorithm traverses the whole tree, which takes $O(V)$, and issues $V_{u}^{D A}$ shortest path queries. The cost to update top-k plateaus is $O(\log k)$ per new plateau found. So, the total cost is $O\left(V+V_{u}^{D A} \times\left(S P_{\text {cost }}+\log k\right)\right)$.

\subsection{Depth-Aware Best First Algorithm}

The depth-aware top down (DA-TD) algorithm processes the backward tree $T_{b}$ in a top down fashion which is suboptimal because many of the top- $k$ plateaus usually appear deeper in the tree. A better approach would access the vertices in the descending order of their maxdepth values. However, maxdepth values of vertices change throughout the algorithm and maintaining a list of vertices sorted on maxdepth is expensive, e.g., for large road networks, the backward tree $T_{b}$ may contain hundreds of thousands of vertices. Next, we propose a novel solution which uses root lists to allow accessing the vertices in the best first order without requiring expensive maintenance.

\subsubsection{Root Lists}

We conceptually partition the backward tree $T_{b}$ into a set of disjoint subtrees. Fig. 5 shows such a conceptual partitioning of the tree $T_{b}$ shown in Fig. 4. Based on the observations presented in Section 3.3.1, we partition $T_{b}$ using two rules: 1) A familiar vertex $v$ and its unfamiliar child $u$ in $T_{b}$ cannot be in the same conceptual partitions (i.e., subtrees) because they cannot be on the same plateau (Lemma 4); 2) Two familiar vertices $u$ and $v$ cannot be in the same subtree if $u$ and $v$ are not on the same plateau (Lemma 5). These two rules can be summarized as follows. Vertex $v$ and its child $u$ are in two disjoint subtrees if $v \cdot \operatorname{depth}[u]=0$. Such instances are represented in Fig. 5 using dashed edges and, note that, each dashed edge connects two disjoint subtrees,

Given the tree $T_{b}$ which is conceptually partitioned into disjoint subtrees as described above, we keep the roots of these subtrees in two separate lists: 1) a sorted root list (denoted as $R L_{S}$ ); and 2) an unsorted root list (denoted as $\left.R L_{U}\right)$. Specifically, we use a threshold $\eta$ and insert every root node $v$ with $v$.maxdepth $>\eta$ in $R L_{S}$ which is kept sorted in descending order of the maxdepth values. Every other root node is pushed to unsorted $R L_{U}$. Consider the example in Fig. 5 and assume that the threshold is $\eta=3$. The sorted root list is $R L_{S}=\left\{v_{6}, v_{5}, v_{8}\right\}$ (sorted on maximum depths 8, 6 and 4 , respectively). The unsorted root list $R L_{U}$ contains all other root nodes not necessarily sorted, i.e., $v_{1}, v_{2}, v_{26}, v_{20}, v_{17}, v_{18}$ and $v_{7}$. Note that the total number of vertices in the backward tree $T_{b}$ is 27 whereas the root list contains 10 roots (equal to the number of conceptual subtree). In our experiments (see Fig. 9(b)), we show that the number of nodes in the sorted root list $R L_{S}$ is around 100 for all road networks even when the backward tree contains up to 1 million nodes. Fig. 9(b) also shows that the unsorted list $R L_{U}$ contains less than $10 \%$ of the nodes in $T_{b}$. Furthermore, in majority of cases, we are able to compute the top- $k$ plateaus by using only the root nodes in $R L_{S}$ whereas $R L_{U}$ is rarely accessed during query processing.

\subsubsection{Maintaining Root Lists}

Recall that a vertex $v$ and its child $u$ are in two different subtrees iff $v$.depth $[u]=0$. Therefore, we only need to handle the situations when $v \cdot \operatorname{depth}[u]$ changes to zero from a non-zero value and when it changes from zero to non-zero.

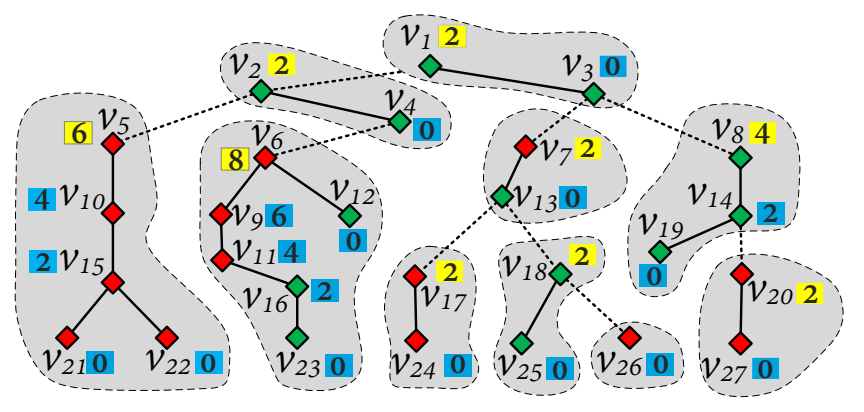

Figure 5: $T_{b}$ with conceptual partitions. Only maxdepth values are shown - depth arrays are omitted for brevity.

Case 1: v.depth $[u]$ becomes zero. If v.depth $[u]$ becomes 0 , this means $u$ and $v$ are now in two different sub-trees (and $u$ is the root of the new subtree). In this case, $u$ is inserted in the root list with u.maxdepth.

Case 2: v.depth[u] becomes non-zero. In this case, $u$ and $v$ were in two different subtrees but now the subtree rooted at $u$ is merged with the subtree that contains $v$. So, $u$ is removed from the root list.

Case 3: v.maxdepth is updated. If $v$ is a root node, its maxdepth is updated in the root list. Furthermore, it is moved from $R L_{S}$ to $R L_{U}$ or vice versa depending on whether $v$.maxdepth is bigger than the threshold $\eta$ or not.

For each vertex $v$ in $T_{b}$, we maintain pointers to their locations in $R L_{S}$ or $R L_{U}$ (the pointer is set to null if the vertex is not in any of the two lists). These pointers allow efficiently updating the root lists.

Note that the above cases can be easily detected and handled in Algorithm 3. In Fig. 5, assume that $v_{1}$ becomes unfamiliar and $v_{10}$ becomes familiar. We call Algorithm 3 by passing to it $v_{10}$ and $v_{1}$. Since these vertices are processed based on level, $v_{10}$ is processed first. As $v_{10}$ is now familiar, computeDepths (Algorithm 2) sets $v_{10}$.depth $\left[v_{15}\right]=0$. This implies that $v_{15}$ is the root of a new subtree. So, $v_{15}$ is inserted in the root list with its maximum depth, i.e., $v_{15} \cdot$ maxdepth $=2$. Since $v_{10}$. maxdepth has changed (to 0) and, as per Algorithm 3, its parent $v_{5}$ is inserted in $L$. In the next iteration, $v_{5}$ is processed. Both $v_{5} . \operatorname{depth}\left[v_{10}\right]$ and $v_{5}$. maxdepth are updated to $v_{10}$. maxdepth $+d\left(v_{10}, v_{5}\right)=$ $0+2=2$. Since $v_{5} \cdot \operatorname{depth}\left[v_{10}\right]$ was non-zero and is still non-zero, this does not indicate a subtree split. However, $v_{5}$. maxdepth is updated from 6 to 2 . Since $v_{5}$ is a root node, its maxdepth is updated in the root list. Assuming $\eta=3, v_{5}$ is moved from $R L_{S}$ to $R L_{U}$. As $v_{5}$.maxdepth has changed, its parent $v_{2}$ is inserted in $L$ and processed but its depth array and maxdepth do not change. Finally, node $v_{1}$ is processed. As $v_{1}$ has now become an unfamiliar node, $v_{1} \cdot \operatorname{depth}\left[v_{2}\right]=v_{2}$. maxdepth $+d\left(v_{2}, v_{1}\right)=2+2=4$. Since $v_{1}$.depth $\left[v_{2}\right]$ was zero and now has become non-zero (Case 2 ), the subtree rooted at $v_{2}$ is merged with the subtree that contains $v_{1}$. Hence, $v_{2}$ is removed from the corresponding root list. Finally, $v_{1}$. maxdepth $=4$ which is updated to 4 in the root list and moved from $R L_{U}$ to $R L_{S}$.

\subsubsection{Algorithm}

The key idea behind our Depth-Aware Best First (DABF) Algorithm (Algorithm 4) is that it traverses each subtree in the sorted root list in the descending order of the maxdepths of their root nodes (lines 2 and 3). Each traversal (line 3 ) is similar to the DA-TD algorithm except that here it does not traverse the subtree of $v$ if $v$ maxdepth $\leq L^{k}$. 
Table 2: Complexity analysis. $V$ is \# of vertices in the shortest path tree. $V_{u}^{T D}$ (resp. $V_{u}^{D A}$ ) is \# of shortest path queries issued by the top-down algorithm (resp. the two depth-aware algorithms). $V_{L}$ is \# of vertices for which maxdepth changes at the timestamp and $V^{>}$is \# of vertices in $T_{b}$ with maxdepth greater than $L^{k}$. $S P_{\text {index }}$ and $S P_{\text {cost }}$ are the index size and cost of the shortest path algorithm used. $E$ is \# of edges in the graph.

\begin{tabular}{|c|c|c|c|c|}
\hline & Tree-Join & Top-Down & Depth-Aware Top-Down & Depth-Aware Best-First \\
\hline Space & $O(E)$ & $O\left(E+S P_{\text {index }}\right)$ & $O\left(E+S P_{\text {index }}\right)$ & $O\left(E+S P_{\text {index }}\right)$ \\
\hline Time & $O(E \log V)$ & $O\left(V+V_{u}^{T D}\left(S P_{\text {cost }}+\log k\right)\right)$ & $O\left(V+V_{u}^{D A}\left(S P_{\text {cost }}+\log k\right)\right)$ & $O\left(V_{L}+V^{>}+V_{u}^{D A}\left(S P_{\text {cost }}+\log k\right)\right)$ \\
\hline
\end{tabular}

Note that it is possible that the unsorted root list $R L_{U}$ contains a top- $k$ plateau if a root node in $R L_{U}$ has maximum depth greater than $L^{k}$. During the update of root lists, we maintain maxdepth $\left(R L_{U}\right)$ which denotes the maximum maxdepth in $R L_{U}$. If maxdepth $\left(R L_{U}\right)>L^{k}$, the algorithm processes the entries in it (line 4). Each entry $e$ is accessed and if e.maxdepth $>\eta$, the entry is moved to $R L_{S}$ (line 6). Also, if e.maxdepth $>L^{k}$, the subtree rooted at $e$ is traversed as described above to update the top- $k$ plateaus and $L^{k}$ (line 8). The algorithm also updates maxdepth $\left(R L_{U}\right)$ (line 9 ) which may have been changed as some entries are moved to $R L_{S}$. Note that the algorithm accesses each unpruned vertex $v$ with maxdepth greater than $L^{k}$ which ensures its correctness.

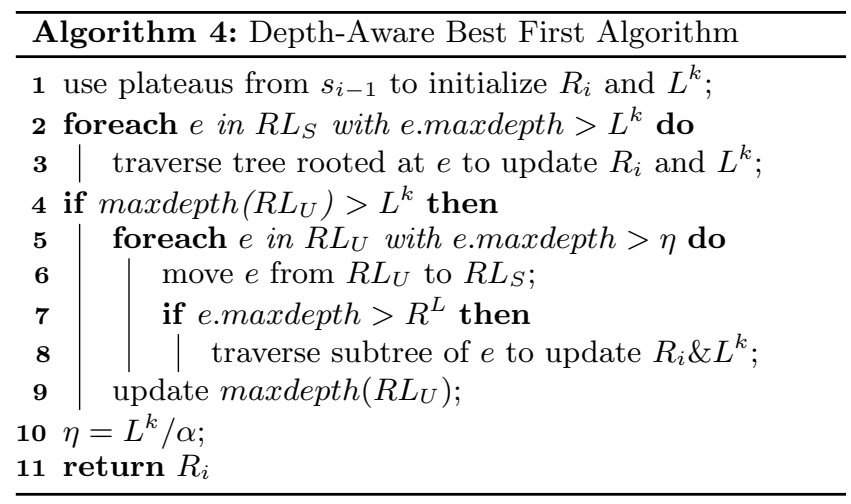

Just before returning the top- $k$ plateaus, we also update $\eta=L^{k} / \alpha$ (line 10), where $\alpha>1$ and helps setting the threshold relative to $L^{k}$. Our experimental study shows that $\alpha=5$ is a reasonable choice. The intuition behind setting $\eta$ several times smaller than $L^{k}$ is to ensure that, in most cases, the top- $k$ plateaus can be found from $R L_{S}$ without the need to process $R L_{U}$. Also, since $L^{k}$ varies throughout the algorithm, $\eta$ also changes. However, unless a root node is implicitly accessed in the root list, we do not move it from $R L_{S}$ to $R L_{U}$ or vice versa. This does not affect the correctness of the algorithm.

Complexity Analysis: Compared to the DA-TD algorithm, the only additional data structure needed by the DA-BF algorithm consists of the two root lists with total size $O(V) \leq O(E)$. Thus, the space complexity is $O\left(E+S P_{\text {index }}\right)$.

Now, we analyse time complexity. As earlier, the cost to maintain maxdepths is $O\left(V_{L}\right)$ where $V_{L}$ is the number of vertices with updated maxdepths. We analyse the cost for maintaining the root lists. Insertion/deletion cost for sorted (resp. unsorted) root list is $O(\log \beta)($ resp. $O(1))$ where $\beta$ is the size of the sorted root list. Note that at most $V_{L}$ entries are inserted/removed in/from the two lists. Let $m$ (resp. $V_{L}-m$ ) be the number of entries inserted/removed in/from the sorted (resp. unsorted) root list. The total maintenance cost is $O\left(V_{L}+m \log \beta+\left(V_{L}-m\right)\right)=O\left(V_{L}+m \log \beta\right)$.
Let $V^{>}$be the number of vertices in $T_{b}$ with maxdepth larger than $L^{k}$. The algorithm traverses at most $V^{>}$vertices during the traversal of all subtrees in the two root lists. Let $\gamma$ be the number of entries in the unsorted list. In the worst case, the algorithm may also access all entries in the unsorted root list (when the results cannot be computed using the sorted list). So, the algorithm accesses $V^{>}+\gamma$ vertices in the worst case. The algorithm issues $V_{u}^{D A}$ shortest path queries where $V_{u}^{D A}$ is the number of unpruned and unmarked vertices (the same as the DA-TD algorithm). Thus, the cost of Algorithm 4 is $O\left(V^{>}+\gamma+V_{u}^{D A} \times\left(S P_{\text {cost }}+\log k\right)\right)$. The total cost including the maintenance of maxdepths and root lists is $O\left(V_{L}+m \log \beta+V^{>}+\gamma+V_{u}^{D A} \times\left(S P_{\text {cost }}+\log k\right)\right)$. Comparison of complexities: Table 2 summarizes the space and time complexities of all the algorithms. For the DA-BF algorithm, we simplify the complexity by removing $\beta, \gamma$ and $m$ because our experimental study shows that $\beta \approx 100$ for all different road networks (Fig. 9(b)). Furthermore, Fig. 9(b) also shows that $\gamma \ll V$ for all road networks. Also, although not shown in the experimental study due to the space limitations, $m<10$ and the unsorted root list, containing $\gamma$ nodes, is rarely accessed by DA-BF (around 1-2 times out of 100). Thus, the cost is simplified to $O\left(V_{L}+V^{>}+V_{u}^{D A} \times\left(S P_{\text {cost }}+\log k\right)\right)$. As shown in Fig. 9(c), $V_{u}^{D A}$ is less than 2 on average, which is significantly smaller than $V_{u}^{T D}$. This explains why the two depth-aware algorithms are significantly faster than the basic top-down (TD) algorithm. Finally, Fig. 9(c) also shows that $V_{L}+V^{>}$is (around 30) which is up to 2 orders of magnitude smaller than $V$. This explains why the DA-BF algorithm outperforms the DA-TD algorithm.

\section{EXPERIMENTS}

\subsection{Settings}

Algorithms and environment. We compare four algorithms that continuously monitor top-k alternative paths, namely the TJ algorithm (Section 3.1), the TD algorithm (Section 3.2), the DA-TD algorithm (Section 3.3), and the DA-BF algorithm (Section 3.4). For the DA-BF algorithm, we studied the effect of $\eta=L^{k} / \alpha$ by using different values of $\alpha$ and observe that the best performance is achieved for $\alpha=5$ which is used as the default value for DA-BF.

Datasets: Table 3 shows details of the eight real road networks in the USA that we use in the experiments (downloaded from DIMACS ${ }^{5}$ ). Similar to some existing works (e.g., see [35]), we fix some issues such as removing self-loops and unconnected components from the graphs.

Parameters: We investigate the effect of varying $k$, length of path $P$, upper bound parameter $\epsilon$, and the road network size. We vary $k$ from 2 to 16 where the default value is 6 . Note that $k=6$ means the shortest path and 5 other alternative paths are reported to the user. Similar to previous

\footnotetext{
${ }^{5}$ http://www.dis. uniroma1.it/challenge9
} 
Table 3: Road Network Datasets

\begin{tabular}{|c|c|c|c|}
\hline Name & Region & \#Vertices & \#Edges \\
\hline NY & New York City & 264,346 & 733,846 \\
\hline COL & Colorado & 435,666 & $1,042,400$ \\
\hline NW & North-west US & $1,207,945$ & $2,545,844$ \\
\hline NE & North-east US & $1,524,453$ & $3,897,636$ \\
\hline E-US & Eastern US & $3,598,623$ & $8,708,058$ \\
\hline W-US & Western US & $6,262,104$ & $15,119,284$ \\
\hline C-US & Central US & $14,081,816$ & $33,866,826$ \\
\hline USA & United States & $23,947,347$ & $57,708,624$ \\
\hline
\end{tabular}

works $[5,25]$ on the snapshot queries, $\epsilon$ is varied from 1.05 to 1.25 and 1.15 is the default value. Alternative paths are continuously reported for each location $s_{i}$ on the user's path and, unless mentioned otherwise, the results report average CPU time for each location, i.e., the total CPU time divided by the number of vertices on the path. For each experiment, we run 1000 queries and present the average cost.

\subsection{Performance Evaluation}

Varying Query Path Length. In this experiment, we vary the distance between source and target of the CTKP query. More specifically, similar to several existing studies on the shortest path queries (e.g., see [34, 27]), we generate eight groups of queries $Q_{1}, Q_{2}, \ldots, Q_{8}$ as follows: we set $l_{\text {min }}$ to $10 \mathrm{~km}$ and set $l_{\max }$ to the maximum distance of any pair of vertices in the road network. Let $z=\left(l_{\max } / l_{\min }\right)^{1 / 8}$. For each $1 \leq i \leq 8$, we generate 1,000 queries to form $Q_{i}$, in which the distance between the source and target vertices for each query pair fall in the range $\left(l_{\min } \times z^{i-1}, l_{\text {min }} \times z^{i}\right]$. Note that the distances of queries increase exponentially in each group from $Q_{1}$ to $Q_{8}$.

The experimental results for CTKP queries in each group from $Q_{1}$ to $Q_{8}$ are shown in Fig. 6 for four different road networks. As expected, the query processing time increases with the increase of the path length, which is mainly because the search space increases with the path length. The TD algorithm performs worst, mainly because, despite pruning, it still requires issuing a large number of shortest path queries. We were not able to run TD for bigger road networks and, hereafter, we omit TD from the comparisons. The two depth-aware algorithms outperform the TJ and TD algorithms by up to several orders of magnitude, especially for bigger road networks. This shows the effectiveness of the ideas proposed in Sections 3.3 and 3.4. Furthermore, DA-BF is several times faster than DA-TD showing the effectiveness of maintaining root lists and accessing the tree in the best first order. Even for the largest road network (USA), DA$\mathrm{BF}$ can continuously monitor $k$ alternative paths in around $1 \mathrm{~ms}$ on average per location $s_{i}$.

Varying k. We vary $k$ and show the results in Fig. 7. The cost of TJ does not change with $k$ because it computes $T_{f}$ and joins it with $T_{b}$ regardless of the value of $k$. The cost of our algorithms increases with increasing $k$ because the algorithms need to compute more plateaus which increases the space searched by our algorithms. Our best algorithm DA-BF is more than two orders of magnitude faster than the TJ algorithm even for the largest $k$. Also, DA-BF is 3-6 times faster than DA-TD, which shows the effectiveness of the proposed best first traversal.

Varying $\epsilon$. In this experiment, we study the effect of the upper bound distance parameter $\epsilon$ and report the results in Fig. 8. As expected, the cost of each algorithm increases with the increase in $\epsilon$ because the search space (i.e., $T_{b}$ ) becomes larger for a larger $\epsilon$. However, DA-BF outperforms TJ by more than two orders of magnitude for all values of $\epsilon$ on bigger road networks. Also, DA-BF is several times faster than DA-TD.

Effectiveness of optimisations. Fig. 9 shows the effectiveness of different optimisations presented in the paper. Specifically, Fig. 9(a) shows the effectiveness of different pruning rules. The No-UB algorithm is the same as DATD except that it does not use the pruning rules that prune the search space using the upper bound distance $d^{P}\left(s_{i}, t\right) \times \epsilon$. Recall that TD uses the Euclidean distance $d^{E}\left(s_{i}, v\right)$ to apply the pruning rules. However, DA-TD uses the exact distance $d\left(s_{i}, v\right)$ if $v$ is a familiar vertex because $d\left(s_{i}, v\right)$ can be obtained in $O(1)$ for the familiar vertices. The No-FUB uses the upper bound pruning rules but does not use this optimisation for the familiar vertices, i.e., it uses Euclidean distance instead of the exact shortest distance for the familiar vertices. As shown in Fig. 9(a), each proposed optimisation significantly improves the performance of the algorithm for different groups of queries $Q_{1}$ to $Q_{8}$.

Fig. 9(b) shows the size of two root lists $R L_{S}$ and $R L_{U}$, the total size of two root lists $R L$ and the total number of valid nodes in $T_{b}$ for different road networks. Note that the size of $R L_{S}$ is around 100 for all road networks. This shows the effectiveness of the root list which maintains only around 100 nodes in sorted order (and the algorithm can find the top- $k$ plateaus using these nodes in most of the cases). Also, the total size of $R L$ is 10 times smaller than the size of $T_{b}$.

Fig. 9(c) shows different variables used in the complexity analysis (Table 2). Recall that $V_{u}^{T D}$ (resp. $V_{u}^{D A}$ ) correspond to the number of shortest path queries issued by TD (resp. DA-TD and DA-BF) per location $s_{i}$. Fig. 9(c) shows that DA-TD and DA-BF need to issue less than 2 shortest path queries per location of $s_{i}$ on average. This shows the effectiveness of maintaining maxdepth values. TD requires issuing up to 1000 shortest path queries on average for each location, which explains the reason why it is slow. Nevertheless, it is able to prune a lot of vertices in $T_{b}$, i.e., $V_{u}^{T D}$ is up to two orders of magnitude smaller than $V$, the size of $T_{b}$. Also, $V_{L}+V^{>}$is significantly smaller than $V$, which explains why DA-BF outperforms DA-TD.

Finally, Fig. 9(d) shows the average number of familiar nodes, unfamiliar nodes and total nodes in $T_{b}$ for each location $s_{i}$ as the user moves on $P$ towards the target. Most of the nodes are familiar, which explains the effectiveness of the depth-aware algorithms. Also, as expected, the size of $T_{b}$ decreases as the user gets closer to the target.

Different road networks (CPU time and memory). Fig. 10 shows the CPU time and memory used by each algorithm for all road networks shown in Table 3. The x-axis shows the road networks in increasing order of their sizes. As expected, Fig. 10(a) shows that the running time of each algorithm increases with the increase in the size of the road network. DA-BF consistently outperforms TJ and DA-TD for all data sets.

The memory used by each algorithm is shown in Fig. 10(b). "Graph" corresponds to the size of the input graph (road network). As expected, DA-BF and DA-TD consume more memory than TJ and TD due to the additional data structures employed (e.g., depth arrays, root lists). However, the memory used by each algorithm increases linearly with the graph size. Recall that the algorithms also need a shortest path index to efficiently compute shortest paths. However, in Fig. 10(b), we do not include the memory used by the shortest path index due to two main reasons: 1) the memory usage depends on the specific shortest path algorithm employed; 2) almost all navigation systems already have a shortest path index which 


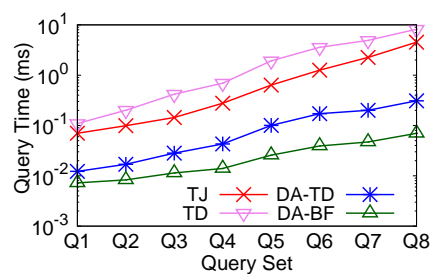

(a) NY

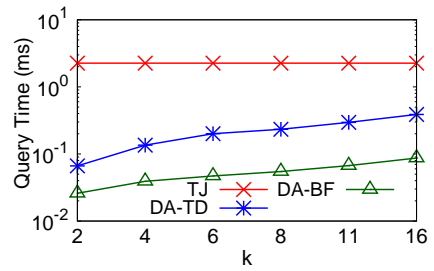

(a) NY

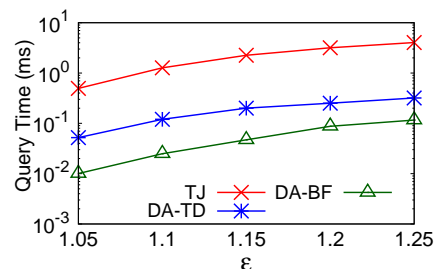

(a) NY

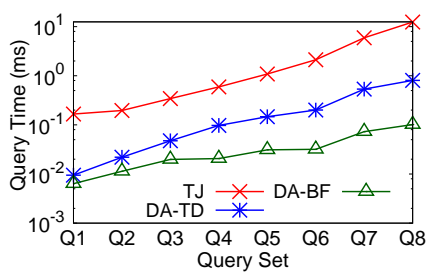

(b) NW

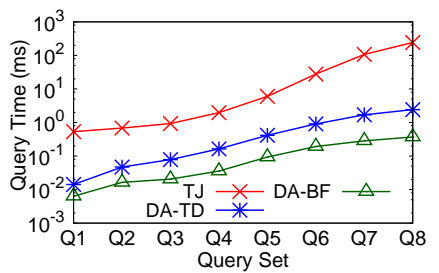

(c) E-US

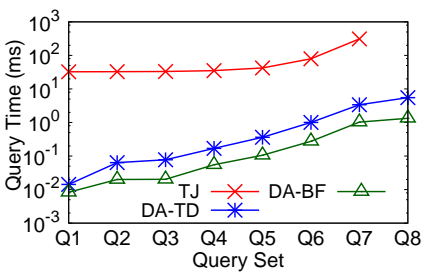

(d) USA

Figure 6: Effect of Varying Path Length

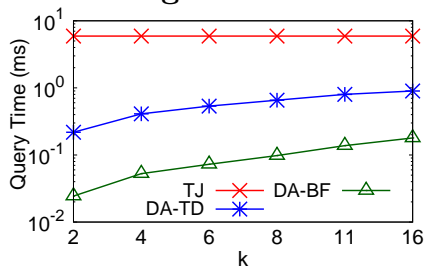

(b) NW

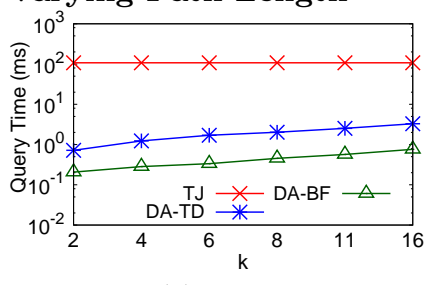

(c) E-US

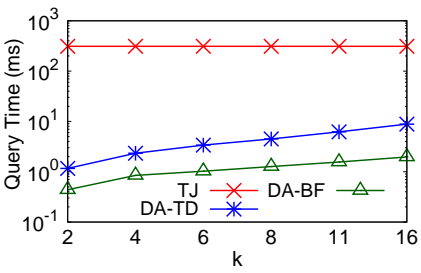

(d) USA

Figure 7: Effect of Varying $k$

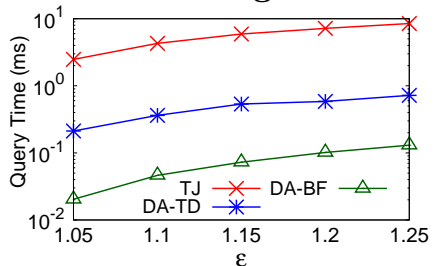

(b) NW

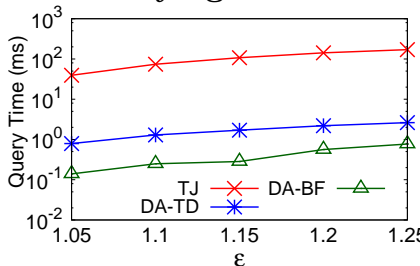

(c) E-US

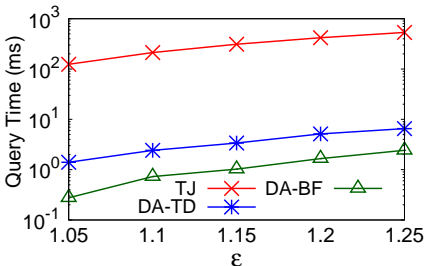

(d) USA

Figure 8: Effect of Varying $\epsilon$

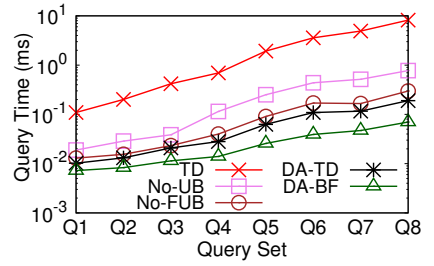

(a) Prune rules

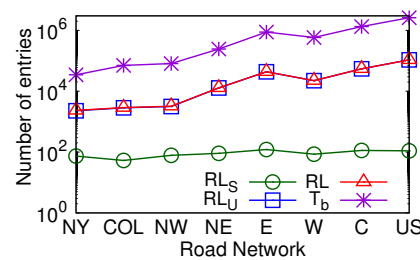

(b) Root list size

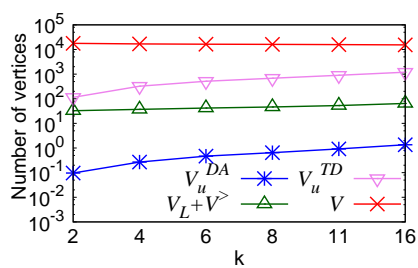

(c) Comp. analysis variables

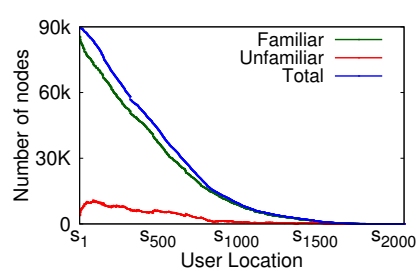

(d) \# of familiar nodes

Figure 9: Effectiveness of optimisations

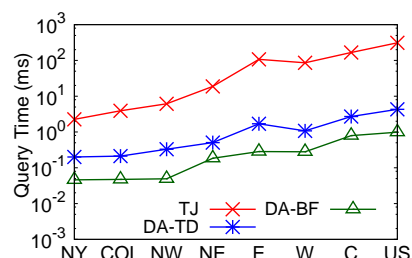

(a) Running time

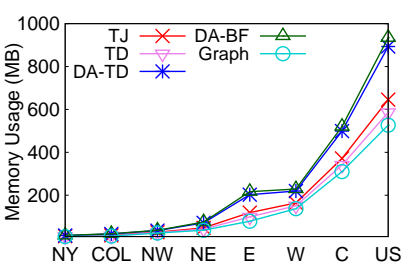

(b) Memory usage
Figure 10: All road networks

our algorithms can directly use (thus Fig. 10(b) represents the memory overhead for each of our algorithms). We used pruned highway labelling (PHL) [7] in our implementation with index size around 16 GB for the US road network as reported in [7] (which dominates the overall memory used by our algorithms). If the index size is an issue, one can use other shortest path algorithms such as Contraction Hierarchies $(\mathrm{CH})$ [18] or Arterial Hierarchy (AH) [37], which have an order of magnitude smaller indexes but provide comparable or better running time for the shortest path queries [23].

\section{CONCLUSIONS AND FUTURE WORK}

To the best of our knowledge, this is the first paper to study the problem of continuously monitoring $k$ alternative paths as the user is moving on a path towards the target. Due to the popularity of plateaus-based alternative paths, we also continuously report alternative paths based on plateaus. We present several algorithms each improving on the previous using some non-trivial observations and novel optimisations. We provide complexity analysis of our algorithms and a comprehensive experimental study using real-world road networks and demonstrate the efficiency of the proposed algorithms. An important direction for future work is to design techniques for snapshot and continuous top- $k$ alternative path queries for dynamic road networks and time-dependent road networks.

Acknowledgements. Muhammad Aamir Cheema is supported by ARC FT180100140 and DP180103411. Hua Lu's work was partly conducted when he was employed at Aalborg University, Denmark. 


\section{REFERENCES}

[1] T. Abeywickrama, M. A. Cheema, and A. Khan. K-SPIN: Efficiently processing spatial keyword queries on road networks. IEEE TKDE, 32(5):983-997, 2019.

[2] T. Abeywickrama, M. A. Cheema, and D. Taniar. K-nearest neighbors on road networks: a journey in experimentation and in-memory implementation. PVLDB, 9(6):492-503, 2016.

[3] I. Abraham, D. Delling, A. V. Goldberg, and R. F. Werneck. A hub-based labeling algorithm for shortest paths in road networks. In International Symposium on Experimental Algorithms. Springer, 2011.

[4] I. Abraham, D. Delling, A. V. Goldberg, and R. F. Werneck. Hierarchical hub labelings for shortest paths. In European Symposium on Algorithms, 2012.

[5] I. Abraham, D. Delling, A. V. Goldberg, and R. F. Werneck. Alternative routes in road networks. Journal of Experimental Algorithmics (JEA), 18:1-3, 2013.

[6] V. Akgün, E. Erkut, and R. Batta. On finding dissimilar paths. European Journal of Operational Research, 121(2):232-246, 2000.

[7] T. Akiba, Y. Iwata, K.-i. Kawarabayashi, and Y. Kawata. Fast shortest-path distance queries on road networks by pruned highway labeling. In ALENEX, pages 147-154, 2014.

[8] R. Bader, J. Dees, R. Geisberger, and P. Sanders. Alternative route graphs in road networks. In International Conference on Theory and Practice of Algorithms in (Computer) Systems, 2011.

[9] F. Barth and S. Funke. Alternative routes for next generation traffic shaping. In Proceedings of the 12th ACM SIGSPATIAL International Workshop on Computational Transportation Science, 2019.

[10] S. Cabello, E. W. Chambers, and J. Erickson. Multiple-source shortest paths in embedded graphs. SIAM Journal on Computing, 42(4):1542-1571, 2013.

[11] M. A. Cheema, L. Brankovic, X. Lin, W. Zhang, and W. Wang. Continuous monitoring of distance-based range queries. IEEE TKDE, 23(8):1182-1199, 2010.

[12] M. A. Cheema, W. Zhang, X. Lin, Y. Zhang, and $\mathrm{X}$. Li. Continuous reverse $\mathrm{k}$ nearest neighbors queries in euclidean space and in spatial networks. The $V L D B$ Journal-The International Journal on Very Large Data Bases, 21(1):69-95, 2012.

[13] Y. Chen, M. G. Bell, and K. Bogenberger. Reliable pretrip multipath planning and dynamic adaptation for a centralized road navigation system. IEEE Trans. on Intelligent Transportation Systems, 2007.

[14] T. Chondrogiannis, P. Bouros, J. Gamper, U. Leser, and D. B. Blumenthal. Finding k-dissimilar paths with minimum collective length. In $A C M$ SIGSPATIAL, pages 404-407, 2018.

[15] T. Chondrogiannis, P. Bouros, J. Gamper, U. Leser, and D. B. Blumenthal. Finding k-shortest paths with limited overlap. The VLDB Journal, pages 1-25, 2020.

[16] E. W. Dijkstra. A note on two problems in connexion with graphs. Numerische mathematik, 1959.

[17] H. Döbler and B. Scheuermann. On computation and application of $\mathrm{k}$ most locally-optimal paths in road networks. 2016.

[18] R. Geisberger, P. Sanders, D. Schultes, and D. Delling. Contraction hierarchies: Faster and simpler hierarchical routing in road networks. In International Workshop on Experimental and Efficient Algorithms, pages 319-333. Springer, 2008.
[19] A. H. Jones. Method of and apparatus for generating routes, Aug. 21 2012. US Patent 8,249,810.

[20] M. Kobitzsch. An alternative approach to alternative routes: Hidar. In European Symposium on Algorithms, pages 613-624. Springer, 2013.

[21] M. Kobitzsch, M. Radermacher, and D. Schieferdecker. Evolution and evaluation of the penalty method for alternative graphs. In ATMOS, 2013.

[22] L. Li, M. A. Cheema, H. Lu, M. E. Ali, and A. N. Toosi. Comparing alternative route planning techniques: A web-based demonstration and user study. arXiv preprint arXiv:2006.08475, 2020.

[23] Y. Li, M. L. Yiu, N. M. Kou, et al. An experimental study on hub labeling based shortest path algorithms. PVLDB, 11(4):445-457, 2017.

[24] H. Liu, C. Jin, B. Yang, and A. Zhou. Finding top-k shortest paths with diversity. IEEE TKDE, 2017.

[25] D. Luxen and D. Schieferdecker. Candidate sets for alternative routes in road networks. Journal of Experimental Algorithmics (JEA), 19:2-7, 2015.

[26] K. Mouratidis, M. L. Yiu, D. Papadias, and N. Mamoulis. Continuous nearest neighbor monitoring in road networks. In Proceedings of the 32nd international conference on Very large data bases, pages 43-54. VLDB Endowment, 2006.

[27] D. Ouyang, L. Qin, L. Chang, X. Lin, Y. Zhang, and Q. Zhu. When hierarchy meets 2-hop-labeling: efficient shortest distance queries on road networks. In SIGMOD, pages 709-724, 2018.

[28] D. Ouyang, L. Yuan, L. Qin, L. Chang, Y. Zhang, and $\mathrm{X}$. Lin. Efficient shortest path index maintenance on dynamic road networks with theoretical guarantees. PVLDB, 13(5):602-615, 2020.

[29] A. Paraskevopoulos and C. D. Zaroliagis. Improved alternative route planning. In ATMOS, 2013.

[30] C. Salgado, M. A. Cheema, and M. E. Ali. Continuous monitoring of range spatial keyword query over moving objects. World Wide Web, 21(3):687-712, 2018.

[31] Z. Shao, M. A. Cheema, D. Taniar, and H. Lu. VIP-tree: An effective index for indoor spatial queries. PVLDB, 10(4):325-336, 2016.

[32] S. Trißl and U. Leser. Fast and practical indexing and querying of very large graphs. In SIGMOD, 2007.

[33] H. Wang, H. He, J. Yang, P. S. Yu, and J. X. Yu. Dual labeling: Answering graph reachability queries in constant time. In ICDE, pages 75-75. IEEE, 2006.

[34] L. Wu, X. Xiao, D. Deng, G. Cong, A. D. Zhu, and S. Zhou. Shortest path and distance queries on road networks: An experimental evaluation. PVLDB, 5(5):406-417, 2012.

[35] B. Yao, Z. Chen, X. Gao, S. Shang, S. Ma, and M. Guo. Flexible aggregate nearest neighbor queries in road networks. In $I C D E$, pages 761-772, 2018.

[36] R. Zhong, G. Li, K.-L. Tan, L. Zhou, and Z. Gong. G-tree: An efficient and scalable index for spatial search on road networks. IEEE TKDE, 2015.

[37] A. D. Zhu, H. Ma, X. Xiao, S. Luo, Y. Tang, and S. Zhou. Shortest path and distance queries on road networks: towards bridging theory and practice. In SIGMOD, 2013. 